\title{
SPECTRAL GAP FOR THE GROWTH-FRAGMENTATION EQUATION VIA HARRIS'S THEOREM*
}

\author{
JOSÉ A. CAÑIZO ${ }^{\dagger}$, PIERRE GABRIEL ${ }^{\ddagger}$, AND HAVVA YOLDAŞ§
}

\begin{abstract}
We study the long-time behavior of the growth-fragmentation equation, a nonlocal linear evolution equation describing a wide range of phenomena in structured population dynamics. We show the existence of a spectral gap under conditions that generalize those in the literature by using a method based on Harris's theorem, a result coming from the study of equilibration of Markov processes. The difficulty posed by the nonconservativeness of the equation is overcome by performing an $h$-transform, after solving the dual Perron eigenvalue problem. The existence of the direct Perron eigenvector is then a consequence of our methods, which prove exponential contraction of the evolution equation. Moreover the rate of convergence is explicitly quantifiable in terms of the dual eigenfunction and the coefficients of the equation.
\end{abstract}

Key words. growth-fragmentation equations, Harris's theorem, spectral gap, long-time behavior of solutions, structured population dynamics

AMS subject classifications. 35B40, 47D06, 92D25, 45K05

DOI. $10.1137 / 20 \mathrm{M} 1338654$

1. Introduction and main result. The growth-fragmentation equation is a linear, partial integro-differential equation which is commonly used in structured population dynamics for modeling various phenomena including the time evolution of cell populations in biology such as in $[1,11,12,22,35,50,57,63,65]$, single species populations [66], or carbon content in a forest [23]; some aggregation and growth phenomena in physics or biophysics as in $[6,28,41,43,55,56]$; neuroscience in [25, 62], and even TCP/IP communication protocols such as in [3, 10,31]. The general form of the growth-fragmentation equation is given by

$$
\begin{array}{rlrl}
\frac{\partial}{\partial t} n(t, x)+\frac{\partial}{\partial x}(g(x) n(t, x))+B(x) n(t, x) & =\int_{x}^{+\infty} \kappa(y, x) n(t, y) \mathrm{d} y, & & t, x>0, \\
n(t, 0) & =0, & t \geq 0 \\
n(0, x) & =n_{0}(x), & & x>0
\end{array}
$$

* Received by the editors May 18, 2020; accepted for publication (in revised form) May 25, 2021; published electronically September 16, 2021.

https://doi.org/10.1137/20M1338654

Funding: The work of the first and third authors was supported by the project MTM201785067-P, funded by the Spanish government and the European Regional Development Fund and they gratefully acknowledge the support of the Hausdorff Research Institute for Mathematics (Bonn), through the Junior Trimester Program on Kinetic Theory. The work of the second author was supported by the ANR project NOLO, grant ANR-20-CE40-0015, funded by the French Ministry of Research. The work of the third author was also supported by the Basque Government through the BERC 2018-2021 program, by the Spanish Ministry of Economy and Competitiveness MINECO: BCAM Severo Ochoa excellence accreditation SEV-2017-0718, by the "la Caixa" Foundation, and by the European Research Council (ERC) under the European Union's Horizon 2020 research and innovation programme grant 639638 .

$\dagger^{\dagger}$ Departamento de Matemática Aplicada, Universidad de Granada, 18071 Granada, Spain (canizo@ugr.es).

${ }_{\ddagger}^{\ddagger}$ Laboratoire de Mathématiques de Versailles, UVSQ, CNRS, Université Paris-Saclay, 45 Avenue des États-Unis, 78035 Versailles Cedex, France (pierre.gabriel@uvsq.fr).

$\S$ Institut Camille Jordan, Université Claude Bernard Lyon 1, Bâtiment Jean Braconnier, 21 Avenue Claude Bernard, 69622 Villeurbanne Cedex, France (yoldas@math.univ-lyon1.fr).

5185

Copyright $@$ by SIAM. Unauthorized reproduction of this article is prohibited. 
where $n(t, x)$ represents the population density of individuals structured by a variable $x>0$ at a time $t \geq 0$. The structuring variable $x$ could be age, size, length, weight, DNA content, biochemical composition, etc., depending on the modeling context. Here we refer to it as "size" for simplicity. Equation (1) is coupled with an initial condition $n_{0}(x)$ at time $t=0$ and a Dirichlet boundary condition which models the fact that no individuals are newly created at size 0 . The function $g$ is the growth rate and $B$ is the total division/fragmentation rate of individuals of size $x \geq 0$. The fragmentation kernel $\kappa(y, x)$ is the rate at which individuals of size $x$ are obtained as the result of a fragmentation event of an individual of size $y$. When fixing $x, \kappa(x, \cdot)$ is a nonnegative measure on $(0, x]$. The total fragmentation rate $B$ is always obtained as

$$
B(x)=\int_{0}^{y} \frac{y}{x} \kappa(x, y) \mathrm{d} y, \quad x>0 .
$$

Important particular cases are

$$
\kappa(x, y)=B(x) \frac{2}{x} \delta_{\left\{y=\frac{x}{2}\right\}},
$$

which corresponds to the mitosis process, suitable for modeling of biological cells, where individuals can only break into two equal fragments; and

$$
\kappa(x, y)=B(x) \frac{2}{x},
$$

which is the case with uniform fragment distribution, where fragmentation gives fragments of any size less than the original one with equal probability. This case is used for example in modeling the dynamics of polymer chains, as in [41].

Two opposing dynamics, growth and fragmentation, are balanced through (1). The growth term tends to increase the average size of the population and the fragmentation term increases the total number of individuals but breaks the population into smaller sizes. If the growth rate $g(x)$ vanishes, then only fragmentation takes place and the equation is known as the pure fragmentation equation. Similarly when $B$ and $\kappa$ are both $0,(1)$ is the pure growth equation.

We are concerned here with the mathematical theory of this equation and, more precisely, with its long-time behavior as $t \rightarrow+\infty$. Under suitable conditions on the coefficients $\kappa$ and $g$, the typical behavior is that the total population tends to grow exponentially at a rate $e^{\lambda t}$ for some $\lambda>0$, and the normalized population distribution tends to approach a universal profile for large times, independently of the initial condition. This has been investigated in a large number of previous works, of which we give a short summary. The first mathematical study of this type of equation was done in [35] for the mitosis case, in a work inspired by some biophysical papers $[11,12,66]$. In [35], the authors considered the mitosis kernel with the size variable in a bounded interval and proved exponential growth at a rate $\lambda$, and exponentially fast approach to the universal profile. In [60], the authors considered the size variable in $(0,+\infty)$ and introduced the general relative entropy method for several linear PDEs including the growth-fragmentation equation. They proved relaxation to equilibrium in $L^{p}$ spaces without an explicit rate. Following [64] and [54], providing an explicit rate of convergence to a universal profile under reasonable assumptions became a topic of research for many other works. New functional inequalities were proved in $[26,27]$ in order to obtain explicit rates of convergence; see also [46]. Some authors used a semigroup approach $[2,5,7,15,40,47,61]$ or a probabilistic approach $[10,16$, 
$17,18,19,20,21,23,29,30,31,32]$, and some authors provided explicit solutions as in [69]. In this paper we are able to give more general results regarding the speed of convergence to equilibrium: we obtain constructive results which cover a wide range of bounded and unbounded fragmentation rates, and which apply both in mitosis and uniform fragmentation situations.

When the equal mitosis kernel is considered, there is a special case with a linear growth rate where the solutions exhibit oscillatory behavior in the long time. This property was first proved mathematically in [47] when the equation is posed in a compact set. Recently, this result was extended to $(0,+\infty)$ by the general relative entropy argument in suitably weighted $L^{2}$ or measure spaces in [13,45] and by means of the Mellin transform in $L^{1}$ space by [68].

An important tool when studying the asymptotic behavior of (1) is the Perron eigenvalue problem: finding a positive eigenfunction for the operator which defines the equation, associated with a simple, real eigenvalue which is also equal to the spectral radius; see [38, 59] for general existence results. In [4], the authors gave some estimates on the principal eigenfunctions of the growth-fragmentation operator, giving their first order behavior close to 0 and $+\infty$. Then they proved a spectral gap result by means of entropy-entropy dissipation inequalities, with tools similar to those of $[26,27]$. They assumed that the growth and the fragmentation coefficients behave asymptotically like power laws.

In this paper we use a probabilistic approach, namely, Harris's theorem, for showing the spectral gap property. We give a novel approach based on estimating solutions to the PDE, and obtain results which can be applied to general growth and fragmentation rates including mitosis and uniform fragmentation cases. Detailed hypotheses and results are given later in this introduction. The method is also completely constructive and gives explicit estimates. However, in some cases these estimates depend on estimates on the first dual eigenfunction, which may be not easy to obtain, but constitute a separate question. After stating our results we also give a brief comparison to other spectral gap results in the literature.

Applications of this type of argument to biological and kinetic models which can be described as Markov processes has become a subject of many works recently, and has been extended to models which are not Markov processes but share similar properties. The predecessor of Harris's theorem, namely, Doeblin's argument is used in [44] for proving exponential relaxation of solutions to the equilibrium for the conservative renewal equation. In [25] and [39], the authors study population models which describe the dynamics of interacting neurons, structured by elapsed time in [25] or by voltage in [39], and the existence of a spectral gap property in the no-connectivity setting is proved by Doeblin's theorem. Moreover, there are some recent works for the extension of this method into the nonconservative setting. In [8], the authors consider several types of linear PDEs including a growth-diffusion model with a timespace varying environment and some renewal equations with time-fluctuating (e.g., periodic) coefficients. They provide quantitative estimates in total variation distance for the associated nonconservative and nonhomogeneous semigroups by means of generalized Doeblin's conditions. The full Harris's theorem is used in [21, 23] for deriving exponential convergence to the equilibrium in the conservative form of the growthfragmentation equation. In the present work, we are interested in the long-time behavior of the more challenging nonconservative case, namely, when no quantity is preserved along time. Our method is in the spirit of [9], where a nonconservative version of Harris's theorem is proposed and applied to the growth-fragmentation equation with constant growth rate $g$ and increasing total division rate $B$; see also [33] for an 
application to a mutation-selection model which is similar to growth-fragmentation. The difference here is that we first build a solution to the dual Perron eigenproblem by using Krein-Rutman's theorem and a maximum principle. Then we take advantage of the dual eigenfunction to perform a so-called (Doob) $h$-transform [36], similarly to $[7,32]$, in order to apply Harris's theorem. It allows us to consider very general growth and fragmentation rates. The drawback is that the spectral gap is given explicitly in terms of the dual eigenfunction, for which quantitative estimates are in general hard to obtain. However, for certain specific coefficients that are worthy of interest, the dual eigenfunction is known explicitly. It is the case of the so-called selfsimilar fragmentation equation, widely studied in the literature, for which we provide new quantitative estimates on the spectral gap.

Let us now make precise the functional analytic setting of our work and what we mean by solutions to (1). We are interested in measure solutions to this equation, which is a relevant notion in population dynamics; see, e.g., [24, 44]. We say that a family $(n(t, \cdot))_{t \geq 0}$ of positive measures on $(0,+\infty)$ is a solution to (1) if for all $f \in C_{c}^{1}([0,+\infty))$ the function $t \mapsto\langle n(t, \cdot), f\rangle$ is continuously differentiable and for all $t \geq 0$

$$
\frac{\mathrm{d}}{\mathrm{d} t}\langle n(t, \cdot), f\rangle=\left\langle n(t, \cdot), \mathcal{L}^{*}[f]\right\rangle
$$

where

$$
\mathcal{L}^{*}[f](x):=g(x) \frac{\partial}{\partial x} f(x)+\int_{0}^{x} \kappa(x, y) f(y) \mathrm{d} y-B(x) f(x)
$$

is the dual operator of the growth-fragmentation operator

$$
\mathcal{L}[n](x):=-\frac{\partial}{\partial x}(g(x) n(x))-B(x) n(x)+\int_{x}^{+\infty} \kappa(y, x) n(y) \mathrm{d} y,
$$

which appears in (1). We refer to [9, 45] for (the method of) proof that (1) is wellposed in the set of positive (or signed Radon) measures $\mu$ such that the weighted total variation norm

$$
\|\mu\|_{V}=\int_{0}^{+\infty} V(x)|\mu|(\mathrm{d} x)
$$

is finite, when $V(x)=x^{k}+x^{K}$ with $k \leq 0$ and $K>1$.

The Perron eigenvalue problem consists of finding suitable eigenelements $(\lambda, N, \phi)$ with $\lambda>0$ and $N, \phi:(0,+\infty) \rightarrow[0,+\infty), N, \phi \not \equiv 0$, satisfying the following:

$$
\begin{gathered}
\mathcal{L}[N]=\lambda N, \quad(g N)(0)=0, \\
\mathcal{L}^{*}[\phi]=\lambda \phi .
\end{gathered}
$$

If such a triple exists then $\lambda$ is actually the dominant eigenvalue of (1), and the solution is expected to converge to a universal profile whose shape is given by the eigenfunction $N(x)$. The convergence rate is given by the gap between the dominant eigenvalue $\lambda>0$ and the rest of the spectrum. If we scale the equation by defining $m(t, x):=n(t, x) e^{-\lambda t}$ we obtain

(7)

$$
\begin{aligned}
\frac{\partial}{\partial t} m(t, x)+\frac{\partial}{\partial x}(g(x) m(t, x))+(B(x)+\lambda) m(t, x) & =\int_{x}^{+\infty} \kappa(y, x) m(t, y) \mathrm{d} y, & & t, x \geq 0, \\
m(t, 0) & =0, & & t>0 \\
m(0, x) & =n_{0}(x), & & x>0 .
\end{aligned}
$$

Copyright (C) by SIAM. Unauthorized reproduction of this article is prohibited. 
We remark that $N(x)$ is the stationary solution of $(7)$ and $\phi(x)$ provides a conservation law for (7) since

$$
\frac{\mathrm{d}}{\mathrm{d} t} \int_{0}^{+\infty} \phi(x) m(t, x) \mathrm{d} x=0 .
$$

Since the existence and uniqueness of the eigenelements provide useful information about the long-time behavior of the growth-fragmentation equation (1), it has been a popular topic of research. We refer to [38] for a general recent result. From now on we consider (7) instead of (1) since it is more convenient to study the long-time behavior of the former and we can easily recover the nature of the latter.

We now list all the assumptions we need throughout the paper.

As we will explain in section 3, Harris's method relies on a local Doeblin's minorization condition. The computations for checking this condition strongly depend on the fragmentation kernel. In [25] a global Doeblin condition is proved (for a similar equation) for kernels $\kappa$ which satisfy, for some $\epsilon, \eta, x_{*}>0$, the condition that $\kappa(x, y) \geq \epsilon$ for all $x \in[0, \eta]$ and $y \geq x_{*}$. Here we rather consider kernels that are of self-similar form, which is commonly assumed in the literature about spectral gaps for the growth-fragmentation equation $[4,9,15,27,29,61]$ and includes the classical kernels appearing in applications (in particular equal or unequal mitosis and uniform fragment distribution; see below).

Hypothesis 1.1. We assume that $\kappa(x, y)$, the fragmentation kernel, is of the selfsimilar form such that

$$
\kappa(x, y)=\frac{1}{x} p\left(\frac{y}{x}\right) B(x) \quad \text { for } y>x>0,
$$

where $p$, the "fragment distribution," is a nonnegative measure on $(0,1]$ such that $z p(z)$ is a probability measure, that is,

$$
\int_{(0,1]} z p(z) \mathrm{d} z=1
$$

Remark 1.1. It is useful to define $p_{k}$ for $k \in \mathbb{R}$, as the $k$ th moment of $p$ :

$$
p_{k}:=\int_{0}^{1} z^{k} p(z) \mathrm{d} z
$$

With this notation, Hypothesis 1.1 ensures that $p_{1}=1$, so the relation (2) is guaranteed.

Our next hypothesis states that we consider only the two extreme cases of the fragment distribution, namely, the very singular equal mitosis case and the very smooth uniform fragment distribution. One can find conditions for our methods to work in intermediate cases, but we have preferred to give simple proofs that show both singular and smooth cases can be treated.

Hypothesis 1.2. We assume that the fragment distribution $p$ is either the one corresponding to the equal mitosis,

$$
p(\mathrm{~d} z)=2 \delta_{\frac{1}{2}}(\mathrm{~d} z)
$$

or the uniform fragment distribution,

$$
p(\mathrm{~d} z)=2 \mathrm{~d} z .
$$


Remark 1.2. We restrict to these two particular fragmentation kernels because they naturally appear in the modeling of natural phenomena. They are also good representatives of two opposite mathematical situations: a very regular, strictly positive case and a singular case which is positive only at $z=1 / 2$. However, the results which we prove to be valid for the uniform kernel can be readily extended to selfsimilar kernels with $p$ satisfying

$$
p(z) \geq c>0 \quad \text { for all } z \text { in some interval }\left(z_{1}, z_{2}\right) \subseteq(0,1)
$$

and either

$$
p_{0}<+\infty \quad \text { if } \quad \int_{0}^{1} \frac{1}{g(x)} \mathrm{d} x<+\infty
$$

or

$$
\exists k<0 \quad \text { with } p_{k}<+\infty \quad \text { if } \quad \int_{0}^{1} \frac{1}{g(x)} \mathrm{d} x=+\infty .
$$

In the particular case of the linear growth rate, $g(x)=x$, it is enough to assume that

$$
\exists k<1 \text { with } p_{k}<+\infty \text {. }
$$

Notice that under condition (10), similarly as for (8) and (9), the function $k \mapsto p_{k}$ is strictly decreasing on the interval where it takes finite values. (The only case in which $p_{k}$ is not strictly decreasing is that of $p(z)$ concentrated at $z=1$, which actually means no fragmentation at all is happening.)

In the case of constant growth rate, a more general condition than (10) is assumed in [9] that covers the unequal mitosis kernels $p(\mathrm{~d} z)=\delta_{\alpha}(\mathrm{d} z)+\delta_{1-\alpha}(\mathrm{d} z)$ with $0<$ $\alpha<1$. In our proofs we can also consider this generalization with straightforward modifications when the growth rate $g$ satisfies forthcoming Hypothesis 1.4.

Regarding non-self-similar kernels, there are results of exponential convergence to the stationary distribution in the literature, but only for bounded fragmentation rates; see [54, 62] for PDE-based arguments and [17, 19, 29] for a probabilistic point of view. We also point out that an optimal condition on the fragment distribution is given in [17] for a spectral gap to exist (for bounded fragmentation rates).

Next we have a general assumption on the growth rate $g$ and the total fragmentation rate $B$.

Hypothesis 1.3 . We assume that $g:(0,+\infty) \rightarrow(0,+\infty)$ is a locally Lipschitz function such that $g(x)=\mathcal{O}(x)$ as $x \rightarrow+\infty$ and $g(x)=\mathcal{O}\left(x^{-\xi}\right)$ as $x \rightarrow 0$ for some $\xi \geq 0$. The total fragmentation rate $B:[0,+\infty) \rightarrow[0,+\infty)$ is a continuous function and the following holds:

$$
\int_{0}^{1} \frac{B(x)}{g(x)} \mathrm{d} x<+\infty, \quad \frac{x B(x)}{g(x)} \underset{x \rightarrow 0}{\longrightarrow} 0, \quad \frac{x B(x)}{g(x)} \underset{x \rightarrow+\infty}{\longrightarrow}+\infty .
$$

This assumption is very mild, and was always present in the previous works to ensure the existence of an equilibrium and a dual eigenfunction. If $B$ behaves like a power of exponent $b$ and $g$ behaves like a power of exponent $a$, conditions (11) are equivalent to the more familiar $b-a+1>0$. The condition $g=\mathcal{O}(x)$ for large $x$ ensures that the characteristics corresponding to the growth part are defined for all times (i.e., clusters do not grow to infinite size in finite time). A stronger assumption which is implicit in Hypothesis 1.3 is that $B$ is bounded above on intervals of the form $[0, R]$ (since it is continuous there), so we do not allow fragmentation rates $B$ which blow up at 0 . This is used in the proof of Lemma 5.1. 
A consequence of Hypothesis 1.3, we will later need the following:

there exists $t_{B}>0$ such that $B$ is bounded below by a positive quantity on any interval of the form $\left[t_{B}, \theta\right]$ with $\theta>t_{B}$.

One sees this from the last limit in (11), which implies that for large enough $t_{B}$ we have

$$
B(x) \geq \frac{g(x)}{x} .
$$

This easily implies (12), since, $g(x) / x$ is continuous and strictly positive, so bounded below by some positive quantity on any compact interval.

Our last assumption gives a stronger requirement on the growth rate $g$ when the mitosis kernel is considered. In this case, some additional requirement is necessary, since, when the linear growth rate with equal mitosis is considered, it is known that there is no spectral gap $[13,45,68]$. We point out that the sharp assumption of "there exists a point $x>0$ with $g(2 x) \neq 2 g(x)$ " is enough to show convergence to the profile $N$ without a rate and only in particular cases, as proved in [65, section 6.3.3]. Our assumption is stronger than this, but also leads to a stronger result.

Hypothesis 1.4. When $p$ is the equal mitosis kernel (8), we assume that the growth rate $g$ satisfies

$$
\begin{gathered}
\omega g(x)<g(\omega x) \quad \text { for all } x>0 \text { and } \omega \in(0,1), \\
H(z):=\int_{0}^{z} \frac{1}{g(x)} \mathrm{d} x<+\infty \quad \text { for all } z>0,
\end{gathered}
$$

and also $H^{-1}$ (the inverse of $H$ ) does not grow too fast, in the sense that for all $r>0$ we have

$$
\lim _{z \rightarrow+\infty} \frac{H^{-1}(z+r)}{H^{-1}(z)}=1 .
$$

If we consider just powers, examples of growth and fragmentation rates which satisfy all of the above are

$$
B(x)=x^{b}, \quad g(x)=x^{a}
$$

with

- any $b \geq 0,-\infty<a \leq 1$ in the uniform fragment distribution case, excluding the case $(b, a)=(0,1)$;

- any $b \geq 0,-\infty<a<1$ in the mitosis case.

Under Hypothesis 1.1, the rescaled growth-fragmentation equation (7) takes the form

$$
\begin{aligned}
\frac{\partial}{\partial t} m(t, x)+\frac{\partial}{\partial x}(g(x) m(t, x))+c(x) m(t, x) & =\mathcal{A}(t, x), & t, x & \geq 0, \\
m(t, 0) & =0, & t & >0, \\
m(0, x) & =n_{0}(x), & & x>0,
\end{aligned}
$$

where

$$
c(x):=B(x)+\lambda
$$

and

$$
\mathcal{A}(t, x):=\int_{x}^{+\infty} \frac{B(y)}{y} p\left(\frac{x}{y}\right) m(t, y) \mathrm{d} y .
$$

According to Hypothesis 1.2 , we only allow $p(z)=2$ or $p(z)=2 \delta_{\frac{1}{2}}(z)$. 
Our main result is given by the following theorem.

TheOREm 1.3. Assume that Hypotheses 1.1, 1.2, 1.3, and 1.4 are satisifed. Then there exists a solution $(\lambda, N, \phi)$ to the Perron eigenvalue problem (5)-(6) with the normalization $\int N=\int \phi N=1, \lambda>0$, and there exist $C, \rho>0$ such that the solution $n=n(t, x) \equiv n_{t}(x)$ to (1) with initial data given by a nonnegative finite measure $n_{0}$ with $\left\|n_{0}\right\|_{V}<+\infty$ satisfies

$$
\left\|e^{-\lambda t} n_{t}-\left(\int \phi n_{0}\right) N\right\|_{V} \leq C e^{-\rho t}\left\|n_{0}-\left(\int \phi n_{0}\right) N\right\|_{V} \quad \text { for all } t \geq 0,
$$

where the weight $V$ of the total variation norm $\|\cdot\|_{V}$ defined in (4) is given by

$$
\begin{aligned}
& V(x)=1+x^{K}, 1+\xi<K \\
& \text { if } \quad \int_{0}^{1} \frac{1}{g(x)} \mathrm{d} x<+\infty \text {, } \\
& V(x)=x^{k}+x^{K},-1<k<0,1+\xi<K \\
& \text { if } \quad \int_{0}^{1} \frac{1}{g(x)} \mathrm{d} x=+\infty .
\end{aligned}
$$

In the specific case of $g(x)=x$, the weight $V(x)$ can be taken to be

$$
V(x)=x^{k}+x^{K},-1<k<1<K .
$$

It is worth noticing that we obtain a spectral gap in spaces with essentially optimal weights. Indeed it was proved in [14] that there is no spectral gap in a weighted $L^{1}$ space with the dual eigenfunction $\phi$ when $B$ is bounded (see the estimates in Theorem 2.1 below).

To the best of our knowledge, even the existence of the Perron eigenelements in such generality is new (allowing a total fragmentation rate with any growth at infinity, and with no required connectivity condition on its support), and hence so is the existence of a spectral gap. However, since our approach for the existence of the principal eigenfunction $N$ is a byproduct of the contraction result provided by Harris's theorem, this precludes the case of self-similar fragmentation with equal mitosis and growth rate $g(x)=x$ for which convergence to a universal profile does not hold, as we already mentioned. In that case the existence of a Perron eigenfunction has to be tackled with other spectral methods, as in [38, 51, 53, 59].

Note also that our result is valid for the measure solutions of (1), thus improving the result in [34] where the general relative entropy method is extended to measure solutions, providing convergence to Malthusian behavior but without a rate and under restrictive assumptions on the coefficients.

Regarding the assumptions on the coefficients, the only existing spectral gap results that consider general growth rates are the ones in [4] and [15]. In these papers, the fragmentation rate is assumed to behave like a power law, which we relax here by only requiring Hypothesis 1.3 on $B$. The other results in the literature focus on constant or linear growth rates and, except in [9], they also consider division rates that grow like power laws.

Finally, when explicit estimates are available for $\phi$, our method allows us to derive quantitative estimates on the spectral gap. It is the case for instance when $g(x)=x$ since then $\phi(x)=x$. An important particular case is to consider additionally that $B(x)=x^{b}$ for some $b>0$. This corresponds to the so-called self-similar fragmentation equation, which appears as a rescaling of the pure fragmentation equation; see, e.g., $[37,42]$. To illustrate the quantification of the spectral gap, we prove that for the 
homogeneous fragmentation kernel and the choice $V(x)=1+x^{2}$, the inequality (15) holds true for

$$
\rho=\frac{-\log \left(1-\frac{\alpha}{2(1+2 \alpha)}\right)}{2 \log 2},
$$

where

$$
\alpha=2 \log 2 R^{b+3} e^{-2(4 R)^{b} / b} \quad \text { with } \quad R=80\left(\frac{15}{2}\right)^{\frac{1}{b}+\frac{b}{2}} .
$$

This seems to simplify the computable bound in [61, Proposition 6.7]. It can also be compared to [46] where the spectral gap in $L^{2}(x \mathrm{~d} x)$ is proved to be at least $\frac{1}{2}$, but only for $b \geq 2$. Similarly as in [61], our method also allows for deriving explicit estimates for more general fragmentation kernels since it does not change the function $\phi$.

Historically, the first explicit spectral gap was obtained for constant growth and division rates and the equal mitosis kernel in [64], and then in [10,31]. The conditions were relaxed [54] and in particular general fragmentation kernels were considered. Our method also allows us to get an explicit spectral gap in the case of constant growth rates, when the division rate is affine and the fragmentation kernel is self-similar. Indeed if $g(x)=1$ and $B(x)=a x+b$, then we easily check that $\phi(x)=\alpha x+1$ with $\alpha=\frac{\left(p_{0}-1\right) b}{2}\left[\sqrt{1+\frac{4 a}{\left(p_{0}-1\right) b^{2}}}-1\right]$, where we recall that $p_{0}$ is the mass of the self-similar kernel $p$, and the Perron eigenvalue is given by $\lambda=\frac{\left(p_{0}-1\right) b}{2}\left[\sqrt{1+\frac{4 a}{\left(p_{0}-1\right) b^{2}}}+1\right]$. It is a particular case of the one treated in [9] where $B$ is only assumed to be nonincreasing, but it extends the historical case of constant division rate.

This paper is organized as follows. We devote section 2 to showing existence of the dual eigenfuction and some bounds on it. In section 3, we recall some introductory concepts from the theory of Markov processes and state Harris's theorem 3.3 based on the previous literature. Eventually for the proof of Theorem 1.3 which is given by applying Harris's theorem, we need to have Hypotheses 3.2 and 3.3 satisfied for (14). In sections 4 and 5, we prove that Hypotheses 3.2 and 3.3 are verified for (14), respectively. Finally in section 6 we give the proof of Theorem 1.3 and the computations leading to (16).

2. Existence of the dual eigenfunction. In this section, we prove the following theorem which implies existence and boundedness of the dual Perron eigenfunction $\phi$, a solution to the dual eigenproblem (6):

ThEOREM 2.1 (existence and bounds on the eigenfunction $\phi$ ). We assume that Hypotheses 1.1 and 1.3 hold true and assume also that $p_{0}<+\infty$. Then there exist a continuous function $\phi$ which is a solution to (6) and $C>0$ such that for any $k>1$,

$$
0<\phi(x) \leq C\left(1+x^{k}\right) \quad \text { for all } x>0 .
$$

Additionally we have $\phi(0)>0$ when $\int_{0}^{1} \frac{1}{g}<+\infty$ and $\phi(0)=0$ when $\int_{0}^{1} \frac{1}{g}=+\infty$.

Notice that our only assumption on $p$ is that $p_{0}<+\infty$ (see Remark 1.2). We prove this theorem at the end of the section.

Following the idea introduced in [64] and also used in [4, 38], we begin with defining a truncated version of the dual Perron eigenproblem (6) in an interval $[0, R]$ 
for some $R>0$ :

$$
\begin{array}{r}
-g(x) \frac{\partial}{\partial x} \phi_{R}(x)+\left(B(x)+\lambda_{R}\right) \phi_{R}(x)=\frac{B(x)}{x} \int_{0}^{R} p\left(\frac{y}{x}\right) \phi_{R}(y) \mathrm{d} y, \\
\phi_{R}(x)>0 \quad \text { for } x \in(0, R), \quad \phi_{R}(R)=0 .
\end{array}
$$

Now we give some lemmas which will be used in the proof of Theorem 2.1. The existence of a solution to (17) is a consequence of the Krein-Rutman theorem (see the appendices in [38] and [4]). Moreover in [4], the authors proved that there exists $R_{0}>0$ large enough such that for all $R>R_{0}$ we have $\lambda_{R}>0$. We thus have the following result.

Lemma 2.2. For any $R>0$, the truncated dual Perron eigenproblem (17) admits a solution $\left(\lambda_{R}, \phi_{R}\right)$ with $\phi_{R}$ a Lipschitz function. Moreover there exists $R_{0}>0$ such that $\lambda_{R}>0$ for all $R>R_{0}$.

Before proving uniform estimates on $\left(\lambda_{R}, \phi_{R}\right)$, we first recall a maximum principle. We begin by defining an operator $\mathcal{L}_{R}^{*}$, acting on once-differentiable functions $\varphi \in$ $\mathcal{C}^{1}([0, R])$ :

$$
\mathcal{L}_{R}^{*} \varphi(x):=-g(x) \varphi^{\prime}(x)+\left(\lambda_{R}+B(x)\right) \varphi(x)-\frac{B(x)}{x} \int_{0}^{x} p\left(\frac{y}{x}\right) \varphi(y) \mathrm{d} y .
$$

We have the following maximum principle; see [38, Appendix C] or [4, section 3.2].

Lemma 2.3. Suppose that $\varphi(x) \geq 0$ for $x \in[0, A]$ for some $A \in(0, R)$ with $\varphi(R) \geq 0$ and $\mathcal{L}_{R}^{*} \varphi(x)>0$ on $[A, R]$. Then $\varphi(x) \geq 0$ on $[0, R]$.

This maximum principle allows us to get a uniform upper bound on $\phi_{R}$ for a suitable normalization.

Lemma 2.4. Consider that Hypotheses 1.1 and 1.3 are satisfied, and that $p_{0}<$ $+\infty$. For any $k>1$, there exists $A>0$ such that if $\phi_{R}$ is normalized such that

$$
\sup _{x \in[0, A]} \phi_{R}(x)=1
$$

then for all $R>\max \left\{A, R_{0}\right\}$ and for all $x \in(0, R]$ we have

$$
0<\phi_{R}(x) \leq 1+x^{k} .
$$

Additionally, $\phi_{R}(0)>0$ when $\int_{0}^{1} \frac{1}{g}<+\infty$ and $\phi_{R}(0)=0$ when $\int_{0}^{1} \frac{1}{g}=+\infty$.

Proof. For the bound from above we want to use the maximum principle in Lemma 2.3. Therefore we want to prove that $\mathcal{L}_{R}^{*} \varphi(x)>0$ for $x \in(A, R)$ with $A \in(0, R)$ as in Lemma 2.3. We take $\varphi(x)=1+x^{k}$ for some $k>1$. Then for $R \geq R_{0}$ we have

$$
\begin{aligned}
\mathcal{L}_{R}^{*} \varphi(x) & =\lambda_{R}\left(1+x^{k}\right)-k g(x) x^{k-1}+B(x)\left(\left(1+x^{k}\right)-\frac{1}{x} \int_{0}^{x}\left(1+y^{k}\right) p\left(\frac{y}{x}\right) \mathrm{d} y\right) \\
& =\lambda_{R}\left(1+x^{k}\right)-k g(x) x^{k-1}+B(x)\left(1+x^{k}-p_{0}-x^{k} p_{k}\right) \\
& >x^{k-1}\left(-k g(x)-B(x) x^{1-k}+\left(1-p_{k}\right) B(x) x\right):=\varrho(x)
\end{aligned}
$$

since $p_{0}=2$ and $0<p_{k}<1=p_{1}$ for $k>1$. Moreover assuming (11) gives that the behavior of $\varrho$ will be dominated by the positive term $\left(1-p_{k}\right) B(x) x^{k}>0$. Therefore, 
we can find $A(k)>0$ such that for all $A(k)<x<R$, we have $\mathcal{L}_{R}^{*} \varphi(x)>0$. We fix such an $A>0$ and normalize $\phi_{R}$ by (18). Then by the maximum principle in Lemma 2.3 we obtain that $\phi_{R}(x) \leq 1+x^{k}$. The positivity or nullity of $\phi_{R}(0)$ is a direct consequence of [4, Theorem 1.10].

LEMma 2.5. Under Hypotheses 1.1 and 1.3 with $p_{0}<+\infty$, there exists a constant $C>0$ such that $\lambda_{R} \leq C$ for all $R>R_{0}$.

Proof. Since $\phi_{R}$ is continuous and by (18), there exists $x_{R} \in[0, A]$ such that $\phi_{R}\left(x_{R}\right)=1$. Notice that necessarily $x_{R}>0$ when $\int_{0}^{1} \frac{1}{g}=+\infty$, since $\phi_{R}(0)=0$ is the case. Moreover, the equation $\mathcal{L}_{R}^{*} \phi_{R}=0$ ensures that for all $x>0$ we have

$$
\begin{aligned}
\left(\phi_{R}(x) \exp \left(-\int_{x_{R}}^{x} \frac{\lambda_{R}+B(s)}{g(s)} \mathrm{d} s\right)\right)^{\prime} & \\
& =-\frac{B(x)}{x g(x)} \exp \left(-\int_{x_{R}}^{x} \frac{\lambda_{R}+B(s)}{g(s)} \mathrm{d} s\right) \int_{0}^{x} p\left(\frac{y}{x}\right) \phi_{R}(y) \mathrm{d} y .
\end{aligned}
$$

By integrating this from $x_{R}$ to $x \geq x_{R}$,

$$
\begin{aligned}
\phi_{R}(x) & \exp \left(-\int_{x_{R}}^{x} \frac{\lambda_{R}+B(s)}{g(s)} \mathrm{d} s\right)-1 \\
& =-\int_{x_{R}}^{x} \frac{B(y)}{y g(y)} \exp \left(-\int_{x_{R}}^{y} \frac{\lambda_{R}+B(s)}{g(s)} \mathrm{d} s\right) \int_{0}^{y} p\left(\frac{z}{y}\right) \phi_{R}(z) \mathrm{d} z \mathrm{~d} y .
\end{aligned}
$$

By using the upper bound on $\phi_{R}$ we obtain, for $R>R_{0}$,

$$
\begin{aligned}
\phi_{R}(x) & \exp \left(-\int_{x_{R}}^{x} \frac{\lambda_{R}+B(s)}{g(s)} \mathrm{d} s\right) \\
& \geq 1-\int_{x_{R}}^{x} \frac{B(y)}{y g(y)} \exp \left(-\int_{x_{R}}^{y} \frac{\lambda_{R}+B(s)}{g(s)} \mathrm{d} s\right) \int_{0}^{y} p\left(\frac{z}{y}\right)\left(1+z^{k}\right) \mathrm{d} z \mathrm{~d} y \\
& \geq 1-\int_{x_{R}}^{x} \frac{B(y)}{g(y)} \exp \left(-\int_{x_{R}}^{y} \frac{\lambda_{R}+B(s)}{g(s)} \mathrm{d} s\right)\left(p_{0}+p_{k} y^{k}\right) \mathrm{d} y .
\end{aligned}
$$

Since $\phi_{R}(R)=0$ we deduce that for all $R>R_{0}$,

$$
\int_{x_{R}}^{R} \frac{B(y)}{g(y)} \exp \left(-\int_{x_{R}}^{y} \frac{\lambda_{R}+B(s)}{g(s)} \mathrm{d} s\right)\left(p_{0}+p_{k} y^{k}\right) \mathrm{d} y \geq 1,
$$

and this enforces $\lambda_{R}$ to be bounded from above. Indeed, otherwise, there would exist a sequence $\left(R_{n}\right)_{n \geq 0}$ and $x_{\infty} \in[0, A]$ such that

$$
R_{n} \rightarrow+\infty, \quad \lambda_{R_{n}} \rightarrow+\infty, \quad x_{R_{n}} \rightarrow x_{\infty} .
$$

But in that case, since

$$
\begin{aligned}
\mathbb{1}_{\left[x_{R_{n}}, R_{n}\right]}(y) \frac{B(y)}{g(y)} & \exp \left(-\int_{x_{R_{n}}}^{y} \frac{\lambda_{R_{n}}+B(s)}{g(s)} \mathrm{d} s\right)\left(p_{0}+p_{k} y^{k}\right) \\
& \leq \frac{B(y)}{g(y)} \exp \left(-\int_{A}^{y} \frac{B(s)}{g(s)} \mathrm{d} s\right)\left(p_{0}+p_{k} y^{k}\right)
\end{aligned}
$$

Copyright $\odot$ by SIAM. Unauthorized reproduction of this article is prohibited. 
and the latter function is integrable on $[0,+\infty$ ) (carry out an integration by parts and use (11)), the dominated convergence theorem ensures that

$$
\int_{x_{R_{n}}}^{R_{n}} \frac{B(y)}{g(y)} \exp \left(-\int_{x_{R_{n}}}^{y} \frac{\lambda_{R_{n}}+B(s)}{g(s)} \mathrm{d} s\right)\left(p_{0}+p_{k} y^{k}\right) \mathrm{d} y \rightarrow 0,
$$

which contradicts (19).

LeMma 2.6. Under Hypotheses 1.1 and 1.3 with $p_{0}<+\infty,\left|\phi_{R}^{\prime}(x)\right|$ is uniformly bounded on compact intervals for all $R>R_{0}$.

Proof. By the equation $\mathcal{L}_{R}^{*} \phi_{R}(x)=0$ and bounds on $\phi_{R}(x)$ and $\lambda_{R}$ we obtain

$$
\begin{aligned}
\left|\phi_{R}^{\prime}(x)\right| & =\frac{\lambda_{R} \phi_{R}(x)}{g(x)}+\frac{B(x)}{g(x)}\left|\phi_{R}(x)-\frac{1}{x} \int_{0}^{x} p\left(\frac{y}{x}\right) \phi_{R}(y) \mathrm{d} y\right| \\
& \leq \frac{\lambda_{R}}{g(x)}\left(1+x^{k}\right)+\frac{B(x)}{g(x)}\left|1+x^{k}-\frac{1}{x}\left(1+x^{k}\right) \int_{0}^{x} p\left(\frac{y}{x}\right) \mathrm{d} y\right| \\
& \leq \frac{\lambda_{R}}{g(x)}\left(1+x^{k}\right)+\frac{B(x)}{g(x)}\left|1+x^{k}-\frac{1}{x}\left(1+x^{k}\right) x p_{0}\right| \\
& \leq \frac{\lambda_{R}}{g(x)}\left(1+x^{k}\right)+\frac{B(x)}{g(x)}\left|1-p_{0}\right|,
\end{aligned}
$$

which gives a bound on $\phi_{R}^{\prime}(x)$ for all $R>R_{0}$, taking into account that $\lambda_{R}$ is uniformly bounded for all $R>R_{0}$ thanks to Lemma 2.5 .

Proof of Theorem 2.1. Lemmas 2.2, 2.4, 2.5, and 2.6 give the proof. Since there exists a solution to the truncated dual Perron eigenproblem (17) for any $R>0$ by Lemma 2.2, it only remains to prove that the terms are bounded in order to pass to the limit as $R \rightarrow+\infty$. We provide the bounds on $\phi_{R}, \lambda_{R}$, and $\phi_{R}^{\prime}$ by Lemmas $2.4,2.5,2.6$, respectively. These bounds ensure that we can extract a subsequence of $\left(\lambda_{R}\right)$ which converges to $\lambda>0$ and a subsequence of $\left(\phi_{R}\right)$ which converges locally uniformly to a limit $\phi$ which satisfies $0<\phi(x) \leq 1+x^{k}$. Clearly $(\lambda, \phi)$ is the solution to the dual Perron eigenproblem (6), and $\phi \not \equiv 0$ since $\sup _{x \in[0, A]} \phi(x)=1$. Similarly, the proof of the positivity or nullity of $\phi(0)$ is a direct consequence of $[4$, Theorem $1.10]$.

3. Harris's theorem. In this section, we state Harris's theorem based on [48] and [49]. The original idea comes from the study of discrete-time Markov processes and dates back to Doeblin and [52] where conditions of existence and uniqueness of having an equilibrium (or an invariant measure) for a Markov process are investigated. It is a probabilistic method which relies on both a minorization property and a drift condition (also called the Foster-Lyapunov condition), which we describe below.

We use Harris's theorem applied to continuous-time Markov processes in order to show that solutions to rescaled growth-fragmentation equation (14), under suitable assumptions, converge towards a universal profile at an exponential rate.

We assume that $\Omega$ is a Polish space and $(\Omega, \Sigma)$ is a measurable space together with its Borel $\sigma$-algebra $\Sigma$, so that $\Omega$ endowed with any probability measure is a Lebesgue space. Moreover we denote the space of finite measures on $\Omega$ by $\mathcal{M}(\Omega)$ and the space of probability measures on $\Omega$ by $\mathcal{P}(\Omega)$.

A discrete-time Markov process $x$ is defined through a transition probability function. A linear, measurable function $S: \Omega \times \Sigma \mapsto \mathcal{P}(\Omega)$ is a transition probability function if $S(x, \cdot)$ is a probability measure for every $x$ and $x \mapsto S(\cdot, A)$ is a measurable 
function for every $A \in \Sigma$. By using the transition probability function we can define the associated Markov operator $\mathcal{S}$ acting on the space of signed measures on $\Omega$ and its adjoint $\mathcal{S}^{*}$ acting on the space of bounded measurable functions $\varphi: \Omega \mapsto[0,+\infty)$ in the following way:

$$
(\mathcal{S} \mu)(A)=\int_{\Omega} S(x, A) \mu(\mathrm{d} x), \quad\left(\mathcal{S}^{*} \varphi\right)(x)=\int_{\Omega} \varphi(y) S(x, \mathrm{~d} y) .
$$

On the other hand, a continuous-time Markov process is no longer described by a single transition function, but by a family of transition probability functions $S_{t}$ defined for each time $t>0$, with the property that the associated operators $\mathcal{S}_{t}$ satisfy

- the semigroup property, $\mathcal{S}_{s+t}=\mathcal{S}_{s} \mathcal{S}_{t}$;

- and $\mathcal{S}_{0}$ is the identity or, equivalently, $S_{0}(x, \cdot)=\delta_{x}$ for all $x \in \Omega$.

We notice that $\mathcal{S}_{t}$ is linear, mass preserving, and positivity preserving. An invariant measure of a continuous-time Markov process $\left(\mathcal{S}_{t}\right)_{t \geq 0}$ is a probability measure $\mu$ on $\Omega$ such that $\mathcal{S}_{t} \mu=\mu$ for every $t \geq 0$, and it is the main concept we need to investigate when studying the asymptotic behavior of a Markov process.

Let us state Doeblin's and Harris's theorems along with some hypotheses. We always assume $\left(\mathcal{S}_{t}\right)_{t \geq 0}$ is a continuous-time Markov semigroup. For their proofs we refer to $[58]$ or $[48,49]$.

Hypothesis 3.1 (Doeblin's condition). There exists a time $t_{0}>0$, a probability distribution $\nu$, and a constant $\alpha \in(0,1)$ such that for any initial condition $x_{0}$ in the domain we have

$$
\mathcal{S}_{t_{0}} \delta_{x_{0}} \geq \alpha \nu
$$

Using this we prove the following theorem.

Theorem 3.1 (Doeblin's theorem). If we have a Markov semigroup $\left(\mathcal{S}_{t}\right)_{t \geq 0}$ satisfying Doeblin's condition (Hypothesis 3.1 ) then for any two finite measures $\mu_{1}$ and $\mu_{2}$ and any integer $n \geq 0$ we have that

$$
\left\|\mathcal{S}_{t_{0}}^{n}\left(\mu_{1}-\mu_{2}\right)\right\|_{\mathrm{TV}} \leq(1-\alpha)^{n}\left\|\mu_{1}-\mu_{2}\right\|_{\mathrm{TV}} .
$$

As a consequence, the semigroup has a unique invariant probability measure $\mu_{*}$, and for all probability measures $\mu$,

$$
\left\|\mathcal{S}_{t}\left(\mu-\mu_{*}\right)\right\|_{\mathrm{TV}} \leq C e^{-\rho t}\left\|\mu-\mu_{*}\right\|_{\mathrm{TV}}, \quad t \geq 0,
$$

where

$$
C:=\frac{1}{1-\alpha}>0, \quad \rho:=\frac{-\log (1-\alpha)}{t_{0}}>0 .
$$

Harris's theorem is an extension of Doeblin's theorem to situations in which one cannot prove a uniform minorization condition as in Hypothesis 3.1. This is often the case when the state space is unbounded. Instead, we use Doeblin's condition only in a given region, and then show that the stochastic process will return to that region often enough. This is established by finding a so-called Lyapunov, or FosterLyapunov function. Both conditions then imply the existence of a spectral gap in a weighted total variation norm. Precisely, we need the following two hypotheses to be satisfied:

Hypothesis 3.2 (Foster-Lyapunov condition). There exist $\gamma \in(0,1), K \geq 0$, some time $t_{0}>0$, and a measurable function $V:[0,+\infty) \mapsto[1,+\infty)$ such that

$$
\left(\mathcal{S}_{t_{0}}^{*} V\right)(x) \leq \gamma V(x)+K
$$

for all $x$. 
Remark 3.2. When our continuous-time Markov process is obtained by solving a particular PDE we often denote

$$
\left(\mathcal{S}_{t} m_{0}\right)(x) \equiv m(t, x),
$$

where $m$ is the solution to the PDE with initial condition $m_{0}$. Then the previous condition is equivalent to

$$
\int_{\Omega} m\left(t_{0}, x\right) V(x) \mathrm{d} x \leq \gamma \int_{\Omega} m_{0}(x) V(x) \mathrm{d} x+K,
$$

to be satisfied for all $m_{0} \in \mathcal{P}(\Omega)$. One can verify this by proving the inequality

$$
\frac{\mathrm{d}}{\mathrm{d} t} \int_{\Omega} m(t, x) V(x) \mathrm{d} x \leq-\lambda \int_{0}^{+\infty} m(t, x) V(x) \mathrm{d} x+D
$$

for some positive constants $D$ and $\lambda$, which then implies (20) with $\gamma=e^{-\lambda t_{0}}$ and $K=D / \lambda$.

The next hypothesis is a minorization condition like Hypothesis 3.1, but only on a sufficiently large region.

Hypothesis 3.3 (small set condition). There exist a probability measure $\nu$, a constant $\alpha \in(0,1)$ and some time $t_{0}>0$ such that

$$
\mathcal{S}_{t_{0}} \delta_{x_{0}} \geq \alpha \nu
$$

for all $x_{0} \in \mathcal{C}$, where

$$
\mathcal{C}=\{x: V(x) \leq R\}
$$

for some $R>2 K /(1-\gamma)$, where $K, \gamma$ are as in Hypothesis 3.2.

Finally we state Harris's theorem under these hypotheses.

TheOREm 3.3 (Harris's theorem). If we have a Markov semigroup $\left(\mathcal{S}_{t}\right)_{t \geq 0}$ satisfying Hypotheses 3.2 and 3.3 then there exist $\beta>0$ and $\bar{\alpha} \in(0,1)$ such that

$$
\left\|\mathcal{S}_{t_{0}} \mu_{1}-\mathcal{S}_{t_{0}} \mu_{2}\right\|_{V, \beta} \leq \bar{\alpha}\left\|\mu_{1}-\mu_{2}\right\|_{V, \beta}
$$

for all nonnegative measures $\int \mu_{1}=\int \mu_{2}$, where the norm $\|\cdot\|_{V, \beta}$ is defined by

$$
\left\|\mu_{1}-\mu_{2}\right\|_{V, \beta}:=\int(1+\beta V(x))\left|\mu_{1}-\mu_{2}\right| \mathrm{d} x .
$$

Moreover, the semigroup has a unique invariant probability measure $\mu_{*}$ and there exist $C>0$ and $\rho>0$ (depending only on $t_{0}, \alpha, \gamma, K, R$, and $\beta$ ) such that

$$
\left\|\mathcal{S}_{t}\left(\mu-\mu_{*}\right)\right\|_{V, \beta} \leq C e^{-\rho t}\left\|\mu-\mu_{*}\right\|_{V, \beta} \text { for all } t \geq 0 .
$$

Explicitly if we set $\gamma_{0} \in[\gamma+2 K / R, 1)$ for any $\alpha_{0} \in(0, \alpha)$ we can choose $\beta=\alpha_{0} / K$ and $\bar{\alpha}=\max \left\{1-\alpha+\alpha_{0},\left(2+R \beta \gamma_{0}\right) /(2+R \beta)\right\}$. Then we have $C=1 / \bar{\alpha}$ and $\rho=$ $-(\log \bar{\alpha}) / t_{0}$.

Proofs of Theorems 3.1 and 3.3 can be found for example in [48, 49, 58, 67].

4. Foster-Lyapunov condition. In this section we prove that Hypothesis 3.2 is verified for the semigroup generated by rescaled growth-fragmentation equation (14), when we consider the evolution of $f(t, x):=\phi(x) m(t, x)$. We divide the proof of Hypothesis 3.2 into three cases which require slightly different calculations.

Copyright (C) by SIAM. Unauthorized reproduction of this article is prohibited. 
4.1. Linear growth rate. First we treat the linear growth case $g(x)=x$ with a constant fragmentation kernel. (As remarked before, we do not consider the mitosis kernel when $g(x)=x$ since there is no spectral gap in that case). In this case the Perron eigenvalue and the corresponding dual eigenfunction are known $(\lambda=1$ and $\phi(x)=x$ ), and the rescaled growth-fragmentation equation is given by

$$
\frac{\partial}{\partial t} m(t, x)+\frac{\partial}{\partial x}(x m(t, x))=2 \int_{x}^{+\infty} \frac{B(y)}{y} m(t, y) \mathrm{d} y-(B(x)+1) m(t, x),
$$

coupled with the usual initial and boundary conditions.

Lemma 4.1. We consider (21) under Hypotheses 1.1, 1.3 with a growth rate $g(x)=x$ and the constant fragment distribution $p(z)=2$ for $z \in(0,1]$. Then the following holds true for any $K>1>k>-1$, for some $C_{1}, \bar{C}>0$, and any nonnegative measure solution $m=m(t, x)$ :

$$
\int_{0}^{+\infty} V(x) f(t, x) \mathrm{d} x \leq e^{-C_{1} t} \int_{0}^{+\infty} V(x) f_{0}(x) \mathrm{d} x+\bar{C} \int_{0}^{+\infty} f_{0}(x) \mathrm{d} x
$$

for all $t \geq 0$, where $f(t, x):=x m(t, x), f_{0}(x)=x m_{0}(x),\left\|f_{0}\right\|_{V}<+\infty$, and $V(x)=$ $x^{k-1}+x^{K-1}$.

Proof. Let $\varphi: \mathbb{R} \rightarrow[0,1]$ be a nonincreasing $C^{1}$ function such that $\varphi(x)=1$ for $x \leq 0$ and $\varphi(x)=0$ for $x \geq 1$. For $\ell>0$ we define $\varphi_{\ell}(x)=\varphi(x-\ell)$. Starting from (3) we have

$$
\begin{aligned}
& \frac{\mathrm{d}}{\mathrm{d} t} \int_{0}^{+\infty}\left(x^{k}+x^{K}\right) \varphi_{\ell}(x) m(t, x) \mathrm{d} x \\
& =\int_{0}^{+\infty}\left(\left(k x^{k-1}+K x^{K-1}\right) \varphi_{\ell}(x)+\left(x^{k}+x^{K}\right) \varphi_{\ell}^{\prime}(x)\right) x m(t, x) \mathrm{d} x \\
& \quad+2 \int_{0}^{+\infty} \frac{B(x)}{x} m(t, x) \int_{0}^{x}\left(y^{k}+y^{K}\right) \varphi_{\ell}(y) \mathrm{d} y \mathrm{~d} x \\
& \quad-\int_{0}^{+\infty}(1+B(x))\left(x^{k}+x^{K}\right) \varphi_{\ell}(x) m(t, x) \mathrm{d} x .
\end{aligned}
$$

Since $\varphi_{\ell}$ is nonincreasing we get

$$
\begin{aligned}
& \frac{\mathrm{d}}{\mathrm{d} t} \int_{0}^{+\infty}\left(x^{k}+x^{K}\right) \varphi_{\ell}(x) m(t, x) \mathrm{d} x \\
& \leq \int_{0}^{+\infty}\left(k x^{k-1}+K x^{K-1}\right) \varphi_{\ell}(x) x m(t, x) \mathrm{d} x \\
& \quad+2 \int_{0}^{+\infty} B(x)\left(\frac{x^{k-1}}{k+1}+\frac{x^{K-1}}{K+1}\right) \varphi_{\ell}(x) x m(t, x) \mathrm{d} x \\
& \quad-\int_{0}^{+\infty}(1+B(x))\left(x^{k-1}+x^{K-1}\right) \varphi_{\ell}(x) x m(t, x) \mathrm{d} x \\
& \leq-\frac{1}{2}(1-k) \int_{0}^{+\infty}\left(x^{k-1}+x^{K-1}\right) \varphi_{\ell}(x) x m(t, x) \mathrm{d} x \\
& \quad+\int_{0}^{+\infty}\left(c_{1} B(x) x^{K-1}+c_{2} x^{K-1}+c_{3} B(x) x^{k-1}+c_{4} x^{k-1}\right) \varphi_{\ell}(x) x m(t, x) \mathrm{d} x,
\end{aligned}
$$

Copyright (c) by SIAM. Unauthorized reproduction of this article is prohibited. 
where

$$
-1<c_{1}:=\frac{1-K}{1+K}<0, \quad c_{2}:=K-\frac{k+1}{2}>0, \quad c_{3}:=\frac{1-k}{1+k}>0, \quad c_{4}:=\frac{k-1}{2}<0 .
$$

We define

$$
\Phi(x):=c_{1} B(x) x^{K-1}+c_{2} x^{K-1}+c_{3} B(x) x^{k-1}+c_{4} x^{k-1} .
$$

Due to Hypothesis 1.3 , the total fragmentation rate $B:[0,+\infty) \rightarrow[0,+\infty)$ satisfies $B(x) \rightarrow 0$ as $x \rightarrow 0$ and $B(x) \rightarrow+\infty$ as $x \rightarrow+\infty$. Hence in the latter expression the behavior as $x \rightarrow+\infty$ is dominated by the first term; thus $\Phi(x)$ will approach $-\infty$. Similarly when $x \rightarrow 0$, the last term will dominate the behavior of $\Phi$, which is negative as well. Since $B$ is continuous we can always bound $\sup _{x \geq 0} \Phi(x) \leq C_{2}$ with some positive quantity $C_{2}>0$. Therefore by denoting $f(t, x)=x m(t, x)$ and $f_{0}(x)=x m_{0}(x)$ we obtain, since $\varphi_{\ell} \leq 1$ and $\int f(t, x) \mathrm{d} x=\int f_{0}(x) \mathrm{d} x$,

$$
\begin{aligned}
& \frac{\mathrm{d}}{\mathrm{d} t} \int_{0}^{+\infty}\left(x^{k-1}+x^{K-1}\right) \varphi_{\ell}(x) f(t, x) \mathrm{d} x \\
& \quad \leq-C_{1} \int_{0}^{+\infty}\left(x^{k-1}+x^{K-1}\right) \varphi_{\ell}(x) f(t, x) \mathrm{d} x+C_{2} \int_{0}^{+\infty} f_{0}(x) \mathrm{d} x
\end{aligned}
$$

where $C_{1}=(1-k) / 2>0$. Then Grönwall's lemma implies

$$
\int_{0}^{+\infty} V(x) \varphi_{\ell}(x) f(t, x) \mathrm{d} x \leq e^{-C_{1} t} \int_{0}^{+\infty} V(x) \varphi_{\ell}(x) f_{0}(x) \mathrm{d} x+\bar{C} \int_{0}^{+\infty} f_{0}(x) \mathrm{d} x
$$

with $\bar{C}=C_{2} / C_{1}$. Due to the monotone convergence theorem we deduce (22) by letting $\ell$ go to $+\infty$.

4.2. Sublinear growth rate close to 0 . In this section we assume that $\int_{0}^{1} \frac{1}{g}<$ $+\infty$, which we sometimes refer to as the case of sublinear growth rate at $x=0$.

LEMMA 4.2. We consider (14) under Hypotheses 1.1, 1.3, and $\int_{0}^{1} \frac{1}{g}<+\infty$. We take $K>1+\xi$. Then the following holds true for $C_{1}=\lambda$ (the first eigenvalue), some $C_{2}>0$, and any nonnegative measure solution $m=m(t, x)$ :

$$
\frac{\mathrm{d}}{\mathrm{d} t} \int_{0}^{+\infty} x^{K} m(t, x) \mathrm{d} x \leq-C_{1} \int_{0}^{+\infty} x^{K} m(t, x) \mathrm{d} x+C_{2} \int_{0}^{+\infty} \phi(x) m(t, x) \mathrm{d} x
$$

for all $t \geq 0$.

Proof. For the sake of conciseness and clarity, we skip the truncation procedure here. But the same method as for Lemma 4.1 can be used to make the calculations rigorous by using the truncation function $\varphi_{\ell}$. We have

$$
\begin{aligned}
\frac{\mathrm{d}}{\mathrm{d} t} & \int_{0}^{+\infty} x^{K} m(t, x) \mathrm{d} x \\
= & -\int_{0}^{+\infty} x^{K} \frac{\partial}{\partial x}(g(x) m(t, x)) \mathrm{d} x-\int_{0}^{+\infty} x^{K}(B(x)+\lambda) m(t, x) \mathrm{d} x \\
& +\int_{0}^{+\infty} x^{K} \int_{x}^{+\infty} \frac{B(y)}{y} p\left(\frac{x}{y}\right) m(t, y) \mathrm{d} y \mathrm{~d} x \\
= & -\lambda \int_{0}^{+\infty} x^{K} m(t, x) \mathrm{d} x+\int_{0}^{+\infty}\left(\left(p_{K}-1\right) x^{K} B(x)+K x^{K-1} g(x)\right) m(t, x) \mathrm{d} x .
\end{aligned}
$$

Copyright (C) by SIAM. Unauthorized reproduction of this article is prohibited. 
We define

$$
\Phi(x):=\left(p_{K}-1\right) x^{K} B(x)+K x^{K-1} g(x)
$$

and notice that $\sup _{x>0} \Phi(x) \leq C_{2} \phi(x)$ for some $C_{2}>0$ due to Hypothesis 1.3 concerning the behavior of $x B(x) / g(x)$ as $x \rightarrow+\infty$ and $x \rightarrow 0$, and the fact that $\phi(0)>0$ since $\int_{0}^{1} \frac{1}{g}<+\infty$ which is a result of Theorem 2.1.

We now give a translation of this lemma in terms of $f=\phi m$, since this is needed in order to apply Harris's theorem to the evolution of $f$.

Corollary 4.3. We consider (14) under Hypotheses 1.1, 1.3, and $\int_{0}^{1} \frac{1}{g}<+\infty$. For $V(x)=1+\frac{x^{K}}{\phi(x)}$, where $K>1+\xi$ and $f(t, x):=\phi(x) m(t, x)$ with $f_{0}(x)=$ $\phi(x) m_{0}(x),\left\|f_{0}\right\|_{V}<+\infty$, there exist $C_{1}, \tilde{C}>0$ such that for all $t \geq 0$

$$
\int_{0}^{+\infty} V(x) f(t, x) \mathrm{d} x \leq e^{-C_{1} t} \int_{0}^{+\infty} V(x) f_{0}(x) \mathrm{d} x+\tilde{C} \int_{0}^{+\infty} f_{0}(x) \mathrm{d} x .
$$

Proof. By adding $\phi(x)$ to both sides of (24) we obtain

$$
\begin{aligned}
& \frac{\mathrm{d}}{\mathrm{d} t} \int_{0}^{+\infty} x^{K} m(t, x) \mathrm{d} x=\frac{\mathrm{d}}{\mathrm{d} t} \int_{0}^{+\infty}\left(x^{K}+\phi(x)\right) m(t, x) \mathrm{d} x \\
& \quad \leq-C_{1} \int_{0}^{+\infty}\left(x^{K}+\phi(x)\right) m(t, x) \mathrm{d} x+\left(C_{1}+C_{2}\right) \int_{0}^{+\infty} \phi(x) m(t, x) \mathrm{d} x .
\end{aligned}
$$

Therefore, we have for $f(t, x)=\phi(x) m(t, x)$,

$$
\begin{aligned}
& \frac{\mathrm{d}}{\mathrm{d} t} \int_{0}^{+\infty}\left(1+\frac{x^{K}}{\phi(x)}\right) f(t, x) \mathrm{d} x \\
& \quad \leq-C_{1} \int_{0}^{+\infty}\left(1+\frac{x^{K}}{\phi(x)}\right) f(t, x) \mathrm{d} x+\left(C_{1}+C_{2}\right) \int_{0}^{+\infty} f_{0}(x) \mathrm{d} x
\end{aligned}
$$

since $\int f(t, x) d x=\int f_{0}(x) d x$. Grönwall's lemma then implies (25) with $\tilde{C}=1+$ $C_{2} / C_{1}$.

4.3. Superlinear growth rate close to $\mathbf{0}$. Now we assume that $\int_{0}^{1} \frac{1}{g}=+\infty$, which implies linear or superlinear behavior for the growth rate $x$ close to 0 . This, of course, includes the case $g(x)=x$ from section 4.1, but the general result we obtain now is slightly more restrictive. In the case of exact linear growth, Lemma 4.1 is slightly more precise.

Lemma 4.4. We consider (14) under Hypotheses 1.1, 1.3, and $\int_{0}^{1} \frac{1}{g}=+\infty$. We take $k<0$ and $K>1+\xi$. Then the following holds true for any nonnegative measure solution $m=m(t, x)$ :

$\frac{\mathrm{d}}{\mathrm{d} t} \int_{0}^{+\infty}\left(x^{k}+x^{K}\right) m(t, x) \mathrm{d} x \leq-C_{1} \int_{0}^{+\infty}\left(x^{k}+x^{K}\right) m(t, x) \mathrm{d} x+C_{2} \int_{0}^{+\infty} \phi(x) m(t, x) \mathrm{d} x$

for all $t \geq 0$, where $C_{1}=\lambda>0$ and $C_{2}>0$ is some constant independent of the solution $m$.

Copyright $@$ by SIAM. Unauthorized reproduction of this article is prohibited. 
Proof. Here again we skip the truncation procedure and refer to the proof of Lemma 4.1 for the method which allows making the calculations rigorous. We have

$$
\begin{aligned}
& \frac{\mathrm{d}}{\mathrm{d} t} \int_{0}^{+\infty}\left(x^{k}+x^{K}\right) m(t, x) \mathrm{d} x \\
&=-\int_{0}^{+\infty}\left(x^{k}+x^{K}\right) \frac{\partial}{\partial x}(g(x) m(t, x)) \mathrm{d} x-\int_{0}^{+\infty}\left(x^{k}+x^{K}\right)(B(x)+\lambda) m(t, x) \mathrm{d} x \\
&+\int_{0}^{+\infty} \frac{B(y)}{y} m(t, y) \int_{0}^{1}\left(y^{k} z^{k}+y^{K} z^{K}\right) p(z) y \mathrm{~d} z \mathrm{~d} y \\
&=-\lambda \int_{0}^{+\infty}\left(x^{k}+x^{K}\right) m(t, x) \mathrm{d} x \\
&+\int_{0}^{+\infty}\left(\left(p_{k}-1\right) x^{k} B(x)+\left(p_{K}-1\right) x^{K} B(x)+k x^{k-1} g(x)+K x^{K-1} g(x)\right) m(t, x) \mathrm{d} x .
\end{aligned}
$$

Similarly to previous proofs, we define

$$
\Phi(x):=\left(p_{k}-1\right) x^{k} B(x)+\left(p_{K}-1\right) x^{K} B(x)+k x^{k-1} g(x)+K x^{K-1} g(x)
$$

and notice that $\sup _{x>0} \Phi(x) \leq C_{2} \phi(x)$ for some $C_{2}>0$ due to Hypothesis 1.3 concerning the behavior of $x B(x) / g(x)$ as $x \rightarrow+\infty$ and $x \rightarrow 0$, and the fact that $p_{K}-1<0$ and $k<0$.

Corollary 4.5. We consider (14) under Hypotheses 1.1, 1.3 and $\int_{0}^{1} \frac{1}{g}=+\infty$. For $V(x)=\frac{x^{k}+x^{K}}{\phi(x)}$ with $k<0, K>1+\xi$, and $f(t, x):=\phi(x) m(t, x)$ with $f_{0}(x)=$ $\phi(x) m_{0}(x),\left\|f_{0}\right\|_{V}<+\infty$, there exist $C_{1}, \tilde{C}>0$ such that for all $t \geq 0$,

$$
\int_{0}^{+\infty} V(x) f(t, x) \mathrm{d} x \leq e^{-C_{1} t} \int_{0}^{+\infty} V(x) f_{0}(x) \mathrm{d} x+\tilde{C} \int_{0}^{+\infty} f_{0}(x) \mathrm{d} x .
$$

Proof. The inequality in Lemma 4.4 yields, for $f(t, x):=\phi(x) m(t, x)$,

$\frac{\mathrm{d}}{\mathrm{d} t} \int_{0}^{+\infty} \frac{x^{k}+x^{K}}{\phi(x)} f(t, x) \mathrm{d} x \leq-C_{1} \int_{0}^{+\infty} \frac{x^{k}+x^{K}}{\phi(x)} f(t, x) \mathrm{d} x+\left(C_{1}+C_{2}\right) \int_{0}^{+\infty} f_{0}(x) \mathrm{d} x$,

since $\int f(t, x) d x=\int f_{0}(x) d x$.

Then Grönwall's lemma implies (26) with $\tilde{C}=1+C_{2} / C_{1}$.

5. Minorization condition. In this section, we show that Hypothesis 3.3 is verified for the semigroup generated by rescaled growth-fragmentation equation (14). We give the proof in two parts where the uniform fragment distribution and the equal mitosis are considered separately.

We start by recalling some known results on the solution of the transport part of (14). Consider the equation

$$
\begin{aligned}
\frac{\partial}{\partial t} m(t, x)+\frac{\partial}{\partial x}(g(x) m(t, x)) & =-c(x) m(t, x), & & t, x>0, \\
m(t, 0) & =0, & & t>0, \\
m(0, x) & =n_{0}(x), & & x>0,
\end{aligned}
$$

which is the same as (14) without the positive part of the fragmentation operator. We remark that Hypothesis 1.3 ensures that the characteristic ordinary differential 
equation

$$
\begin{aligned}
\frac{\mathrm{d}}{\mathrm{d} t} X_{t}\left(x_{0}\right) & =g\left(X_{t}\left(x_{0}\right)\right), \\
X_{0}\left(x_{0}\right) & =x_{0},
\end{aligned}
$$

has a unique solution, defined for $t \in[0,+\infty)$, for any initial condition $x_{0}>0$. In fact, it is defined in some interval $\left(t_{*}\left(x_{0}\right),+\infty\right)$ for some $t_{*}\left(x_{0}\right)<0$. The solution can be explicitly given in terms of $H^{-1}$, where

$$
H(x):=\int_{1}^{x} \frac{1}{g(y)} \mathrm{d} y, \quad x \geq 0 .
$$

We notice that $H$ is strictly increasing with $H_{0}:=H(0)=\lim _{x \rightarrow 0} H(x)<0$ and $\lim _{x \rightarrow+\infty} H(x)=+\infty$ (since $g$ grows sublinearly as $x \rightarrow+\infty$ ), so that it is invertible as a map from $(0,+\infty)$ to $\left(H_{0},+\infty\right)$. (We allow $H_{0}=-\infty$ if $1 / g$ is not integrable close to $x=0$.) It can easily be checked that

$$
X_{t}\left(x_{0}\right)=H^{-1}\left(t+H\left(x_{0}\right)\right) \quad \text { for } x_{0}>0 \text { and } t>H_{0}-H\left(x_{0}\right),
$$

so that that the maximal time interval where the solution of (28) is defined is precisely as $\left(H_{0}-H\left(x_{0}\right),+\infty\right)$. Since it will be convenient later, we define

$$
X_{t}(0):=\lim _{x_{0} \rightarrow 0} X_{t}\left(x_{0}\right)= \begin{cases}0 & \text { if } H_{0}=-\infty \\ H^{-1}\left(t+H_{0}\right) & \text { if } H_{0} \in(-\infty, 0) .\end{cases}
$$

This reflects the fact that the characteristics take a very long time to escape from 0 when $1 / g$ is not integrable close to 0 ; while they escape in finite time if $1 / g$ is integrable close to 0 . For each $t \geq 0$, we have thus defined the flow map $X_{t}:(0,+\infty) \rightarrow$ $\left(X_{t}(0),+\infty\right)$, which is strictly increasing. For negative times, we may consider $X_{-t}:\left(X_{t}(0),+\infty\right) \rightarrow(0,+\infty)$ (where $\left.t>0\right)$. Of course, $X_{-t}=\left(X_{t}\right)^{-1}$.

If $n_{0}$ is a nonnegative measure, it is well known that the unique measure solution to (27) is given by

$$
\begin{array}{ll}
m(t, x)=X_{t} \# n_{0}(x) \exp \left(-\int_{0}^{t} c\left(X_{-\tau}(x)\right) \mathrm{d} \tau\right), & t \geq 0, x>X_{t}(0), \\
m(t, x)=0, & t \geq 0, x \leq X_{t}(0),
\end{array}
$$

where we abuse notation by evaluating the measures $m(t, \cdot)$ and $X_{t} \# n_{0}$ at a point $x>0$. For a Borel measurable map $X:(0,+\infty) \rightarrow(0,+\infty)$, the expression $X \# n_{0}$ denotes the transport, or push forward, of the measure $n_{0}$ by the map $X$, defined by duality through

$$
\int_{0}^{\infty} \varphi(x) X \# n_{0}(x) \mathrm{d} x:=\int_{0}^{\infty} \varphi(X(y)) n_{0}(y) \mathrm{d} y
$$

for all continuous, compactly supported $\varphi:(0,+\infty) \rightarrow \mathbb{R}$. We use the notation $\mathcal{T}_{t}$ for this flow map:

$$
\mathcal{T}_{t} n_{0}(x):=X_{t} \# n_{0}(x) \quad \text { for all } t \geq 0,
$$

so $\mathcal{T}_{t}$ is the semigroup associated with transport equation (27). 
If additionally $n_{0}$ is a function and $X$ has a left inverse $X^{-1}:(a, b) \rightarrow(0,+\infty)$, one has

$$
X \# n_{0}(x)= \begin{cases}\left.n_{0}\left(X^{-1}(x)\right) \mid \frac{\mathrm{d}}{\mathrm{d} x}\left(X^{-1}\right)(x)\right) \mid & \text { if } x \in(a, b), \\ 0 & \text { otherwise. }\end{cases}
$$

Using this for the solution to (27), if $n_{0}$ is a function we may write $m$ in the equivalent form

$$
m(t, x)=n_{0}\left(X_{-t}(x)\right) \frac{\mathrm{d}}{\mathrm{d} x} X_{-t}(x) \exp \left(-\int_{0}^{t} c\left(X_{-\tau}(x)\right) \mathrm{d} \tau\right)
$$

when $t \geq 0$ and $x>X_{t}(0)$, and $m(t, x)=0$ otherwise. Using that $Y_{t}(x):=\frac{\mathrm{d}}{\mathrm{d} x} X_{t}(x)$ satisfies $\frac{\mathrm{d}}{\mathrm{d} t} Y_{t}(x)=g^{\prime}\left(X_{t}(x)\right) Y_{t}(x)$, we note for later that

$$
\frac{\mathrm{d}}{\mathrm{d} x} X_{-t}(x)=\exp \left(-\int_{0}^{t} g^{\prime}\left(X_{-\tau}(x)\right) \mathrm{d} \tau\right), \quad t \geq 0, x>X_{t}(0) .
$$

5.1. Uniform fragment distribution. Let us consider the case of uniform fragment distribution $p(z)=2$, corresponding to the fragmentation kernel of the form $\kappa(x, y)=\frac{2}{x} B(x) \mathbb{1}_{\{0 \leq x \leq y\}}$. The growth-fragmentation equation in this case is widely studied and depending on some assumptions made on growth and total division rates, existence (in some cases exact values) of eigenelements are known. The rescaled growth-fragmentation equation in this case becomes

$$
\begin{aligned}
\frac{\partial}{\partial t} m+\frac{\partial}{\partial x}(g(x) m) & =2 \int_{x}^{+\infty} \frac{B(y)}{y} m(t, y) \mathrm{d} y-(B(x)+\lambda) m, & & t, x \geq 0, \\
m(t, 0) & =0, & & t>0, \\
m(0, x) & =n_{0}(x), & & x>0,
\end{aligned}
$$

where $m=m(t, x)$ whenever variables are not explicitly written. If we consider a linear growth $g(x)=g_{0} x$ and a power like total division $B(x)=b_{0} x^{\gamma}$ with $\gamma>0$, and $g_{0}, b_{0}>0$, the Perron eigenvalue and the corresponding dual eigenfunction are given by

$$
\lambda=g_{0} \quad \text { and } \quad \phi(x)=\frac{x}{\int y N(y)} .
$$

In this case, eigenelements can be computed explicitly (see, for example, [38]):

$$
\lambda=g_{0}, \quad N(x)=\left(\frac{b_{0}}{\gamma g_{0}}\right)^{1 / \gamma} \frac{\gamma}{\Gamma\left(\frac{1}{\gamma}\right)} \exp \left(-\frac{1}{\gamma} \frac{b_{0}}{g_{0}} x^{\gamma}\right), \quad \phi(x)=\left(\frac{b_{0}}{\gamma g_{0}}\right)^{1 / \gamma} \frac{\Gamma\left(\frac{1}{\gamma}\right)}{\Gamma\left(\frac{2}{\gamma}\right)} x .
$$

Moreover, in [4], the authors give the asymptotics of the profile $N$ and accurate bounds on the dual eigenfunction $\phi$ in a more general form of the growth-fragmentation equation, where growth and total division rates behave like a power law for large and small $x$.

Lemma 5.1 (lower bound for the uniform fragment distribution). Assume Hypotheses 1.1 and 1.3 hold true with a constant distribution of fragments $p(z)=2$ for $z \in(0,1]$. Let $\left(\mathcal{S}_{t}\right)_{t \geq 0}$ be the linear semigroup associated with (33). For all $0<\eta<\theta$ 
given, there exists $t_{0}>0$ such that for all $t>t_{0}$ and $x_{0} \in(\eta, \theta]$ it holds that

$$
\mathcal{S}_{t} \delta_{x_{0}}(x) \geq C(\eta, \theta, t) \quad \text { for all } x \in I_{t},
$$

where $I_{t}$ is an open interval which depends on $\eta$, the time $t$, and for some quantity $C=C(\eta, \theta, t)$ depending only on $\eta, \theta$, and $t$. If in addition we assume that

$$
\int_{0}^{1} \frac{1}{g(x)} \mathrm{d} x<+\infty
$$

then the above result also holds when taking $\eta=0$.

Proof. Recall that $\left(\mathcal{T}_{t}\right)_{t \geq 0}$ is the semigroup associated with the transport equation

$$
\frac{\partial}{\partial t} m(t, x)+\frac{\partial}{\partial x}(g(x) m(t, x))+c(x) m(t, x)=0,
$$

where $c(x)=B(x)+\lambda$. By Duhamel's formula we have

$$
\mathcal{S}_{t} n_{0}(x)=m(t, x)=\mathcal{T}_{t} n_{0}(x)+\int_{0}^{t} \mathcal{T}_{t-\tau}(\mathcal{A}(\tau, .))(x) \mathrm{d} \tau,
$$

where $\mathcal{A}(t, x):=2 \int_{x}^{+\infty} \frac{B(y)}{y} m(t, y) \mathrm{d} y$. Fix $0 \leq \eta<\theta$, and take any $x_{0} \in(\eta, \theta]$.

If $n_{0}=\delta_{x_{0}}$, a simple bound gives

$$
\mathcal{S}_{t} \delta_{x_{0}} \geq \mathcal{T}_{t} \delta_{x_{0}}=X_{t} \# \delta_{x_{0}} \exp \left(-\int_{0}^{t} c\left(X_{t-\tau}\left(x_{0}\right)\right) \mathrm{d} \tau\right)
$$

where we have used the expression of $\mathcal{T}_{t}$ given in (29) and the fact that the support of $X_{t} \# \delta_{x_{0}}$ is the single point $\left\{X_{t}\left(x_{0}\right)\right\}$. By Hypothesis 1.3 (in particular since $B$ is continuous on $\left.\left[0, X_{t}(\theta)\right]\right)$, for some $C_{1}=C_{1}(\theta, t)$ which is increasing in $t$, we have

$$
c(x)=B(x)+\lambda \leq C_{1} \quad \text { for all } x \in\left(0, X_{t}(\theta)\right] .
$$

We deduce that

$$
\mathcal{S}_{t} \delta_{x_{0}} \geq X_{t} \# \delta_{x_{0}} e^{-C_{1} t}=\delta_{X_{t}\left(x_{0}\right)} e^{-C_{1} t} .
$$

Using this we obtain

$$
\mathcal{A}(t, x) \geq 2 e^{-C_{1} t} \frac{B\left(X_{t}\left(x_{0}\right)\right)}{X_{t}\left(x_{0}\right)} \quad \text { for all } t>0 \text { and } x<X_{t}\left(x_{0}\right) .
$$

We use that there is some $x_{B}>0$ for which $B$ is bounded below by a positive quantity on any interval of the form $\left[x_{B}, R\right]$. There is some $t_{B}>0$ such that for $t>t_{B}$ we have $X_{t}\left(x_{0}\right)>x_{B}$ for all $x_{0}>\eta$ (for this to hold, notice we may take $\eta=0$ in the case that $\int_{0}^{1} 1 / g<+\infty$, but we need $\eta>0$ otherwise). Hence, for some $C_{2}=C_{2}(\eta, \theta, t)$ which is decreasing in $t$, we obtain

$$
\mathcal{A}(t, x) \geq C_{2} e^{-C_{1} t} \quad \text { for all } t>t_{B} \text { and } x<X_{t}\left(x_{0}\right) .
$$

Take now $t>t_{B}$, which will stay fixed until the end of the proof. The previous bound shows that

$$
\mathcal{A}(\tau, x) \geq C_{2}(\eta, \theta, \tau) e^{-C_{1}(\theta, \tau) \tau} \geq C_{2}(\eta, \theta, t) e^{-C_{1}(\theta, t) \tau}=: \tilde{C}_{2} e^{-\tilde{C_{1} \tau}}
$$

Copyright $@$ by SIAM. Unauthorized reproduction of this article is prohibited. 
for all $t>t_{B}, t_{B}<\tau<t$, and all $x<X_{\tau}\left(x_{0}\right)$. As a consequence, using (31) and (32),

$$
\mathcal{T}_{t-\tau} \mathcal{A}(\tau, x) \geq \tilde{C_{2}} e^{-\tilde{C_{1} \tau}} \exp \left(-\int_{0}^{t-\tau} c\left(X_{-s}(x)\right) \mathrm{d} s\right) \exp \left(-\int_{0}^{t-\tau} g^{\prime}\left(X_{-s}(x)\right) \mathrm{d} s\right)
$$

for all $t_{B}<\tau<t$ and $X_{t-\tau}(0)<x<X_{t}\left(x_{0}\right)$. Since $X_{-s}(x) \leq X_{t}\left(x_{0}\right)$ in this range, we can bound this by

$$
\mathcal{T}_{t-\tau} \mathcal{A}(\tau, x) \geq \tilde{C}_{2} e^{-2 \tilde{C_{1} t}} \exp \left(-\int_{0}^{t-\tau} g^{\prime}\left(X_{-s}(x)\right) \mathrm{d} s\right),
$$

again for all $t_{B}<\tau<t$ and $X_{t-\tau}(0)<x<X_{t}\left(x_{0}\right)$. In order to find a lower bound for the last exponential we restrict to a smaller $x$ interval. Since the bound holds for all $x$ with

$$
X_{t-\tau}(0)<x<X_{t}\left(x_{0}\right),
$$

it holds in particular for all $x$ with

$$
X_{t-t_{B}}(\eta)<x<X_{t}(\eta) .
$$

Again this is a point where we need to take $\eta>0$ in the case $\int_{0}^{1} 1 / g=+\infty$, since otherwise this gives an empty range of $x$. In the case $\int_{0}^{1} 1 / g<+\infty, \eta=0$ is allowed. In this range, the quantity $X_{-s}(x)$ inside the exponential satisfies

$$
X_{\tau-t_{B}}(\eta) \leq X_{-s}(x) \leq X_{t}(\eta) .
$$

Choose $\delta>0$ such that $t_{B}+\delta<t$. Then for all $x$ satisfying (35) and all $\tau \in\left(t_{B}+\delta, t\right)$ we have

$$
X_{\delta}(\eta) \leq X_{-s}(x) \leq X_{t}(\eta)
$$

Using that $g^{\prime}(X) \leq C_{3}$ for all $X \in\left[X_{\delta}(\eta), X_{t}(\eta)\right]$ we have

$$
\mathcal{T}_{t-\tau} \mathcal{A}(\tau, x) \geq \tilde{C_{2}} e^{-\tilde{C_{1} \tau}} e^{-C_{3}(t-\tau)} \geq \tilde{C_{2}} e^{-C_{4} t}
$$

for all $x$ satisfying (35) and all $\tau \in\left(t_{B}+\delta, t\right)$. A final integration gives, for $x$ in the same interval,

$$
\int_{0}^{t} \mathcal{T}_{t-\tau}(\mathcal{A}(\tau, \cdot))(x) \mathrm{d} \tau \geq \tilde{C}_{2} e^{-C_{4} t} \int_{t_{B}+\delta}^{t} \mathrm{~d} \tau=\tilde{C}_{2} e^{-C_{4} t}\left(t-t_{B}-\delta\right) .
$$

Taking $t_{0}:=t_{B}$ gives the result.

5.2. Equal mitosis. We now consider the fragment distribution $p(z)=2 \delta_{\frac{1}{2}}(z)$ which describes the process of equal mitosis, in which cells of size $x$ split into two equal daughter cells of size $x / 2$. In (14), we then have $\mathcal{A}(t, x):=4 B(2 x) m(t, 2 x)$ and the rescaled growth-fragmentation equation takes the form

$$
\begin{aligned}
\frac{\partial}{\partial t} m(t, x)+\frac{\partial}{\partial x}(g(x) m(t, x)) & =4 B(2 x) m(t, 2 x)-(B(x)+\lambda) m(t, x), & & t, x \geq 0 \\
m(t, 0) & =0, & & t>0 \\
m(0, x) & =n_{0}(x), & & x>0
\end{aligned}
$$

Copyright (c) by SIAM. Unauthorized reproduction of this article is prohibited. 
The case where $g$ and $B$ are constant was the subject of numerous works in the past, most notably $[10,31,50,61,64,68]$. For $g(x)=1$ and $B(x)=1$, eigenelements are given by

$$
\lambda=1, \quad N(x)=\sum_{n=0}^{+\infty}(-1)^{n} \alpha_{n} e^{-2^{n+1} x}, \quad \phi(x) \equiv 1,
$$

with $\alpha_{n}=\frac{2}{2^{n}-1} \alpha_{n-1}$ and $\alpha_{0}>0$ a suitable normalization constant, and the solution $m(t, x)$ converges exponentially fast to the universal profile $N(x)$, which vanishes as $x \rightarrow 0$ and $x \rightarrow+\infty$. However, when a linear growth rate $g(x)=x$ is considered (36) exhibits oscillatory behavior in the long-time. This is because instead of a dominant real eigenvalue, there are nonzero imaginary eigenvalues, so that there exists a set of dominant eigenvalues. This type of periodic long-time behavior was first observed in [35] and then it was proved in [47] by using the theory of positive semigroups combined with spectral analysis to obtain the convergence to a semigroup of rotations. Since the method relies on some compactness arguments, the authors considered the equation in a compact subset of $(0,+\infty)$. Recently in [45], the authors proved the oscillatory behavior in the framework of measure solutions for general division rates on $(0,+\infty)$. The proof relies on a general relative entropy argument combined with the use of Harris's theorem on discrete subproblems. It provides an explicit rate of convergence in the weighted total variation norm. Here we consider a sublinear growth rate and a more general division rate than those so far considered in the literature. We exclude of course the case $g(x)=x$, for which we know the lower bound (and the exponential convergence) does not hold.

We first need a technical lemma which gives an expression for the time integration of a measure moving in time.

Lemma 5.2. Let $t>0$ and $F:[0, t] \rightarrow \mathbb{R}$ an injective, differentiable function. Then

$$
\int_{0}^{t} \delta_{F(\tau)}(x) \mathrm{d} \tau=\left(F^{-1}\right)^{\prime}(x) \mathbb{1}_{\{F(0) \leq x \leq F(t)\}} .
$$

Proof. Integrating against a smooth test function $\varphi(x)$ we obtain

$$
\begin{aligned}
\int_{0}^{+\infty} \varphi(x) \int_{0}^{t} \delta_{F(\tau)}(x) \mathrm{d} \tau \mathrm{d} x & =\int_{0}^{t} \int_{0}^{+\infty} \varphi(x) \delta_{F(\tau)}(x) \mathrm{d} x \mathrm{~d} \tau \\
& =\int_{0}^{t} \varphi(F(\tau)) \mathrm{d} \tau=\int_{F(0)}^{F(t)} \varphi(y)\left(F^{-1}\right)^{\prime}(y) \mathrm{d} y
\end{aligned}
$$

by using the change of variable $y=F(\tau)$.

The following result will ensure a certain sublinearity of the characteristic flow $X_{t}$ which we will need later:

Lemma 5.3. Assume that the growth rate $g:(0,+\infty) \rightarrow(0,+\infty)$ is locally Lipschitz and satisfies

$$
\omega g(x)<g(\omega x) \quad \text { for all } x>0 \text { and } \omega \in(0,1) .
$$

Then for any $t>0$ the characteristic flow $X_{t}$ satisfies

$$
\omega X_{t}(x)<X_{t}(\omega x) \quad \text { for all } x>0 \text { and } \omega \in(0,1) .
$$

Copyright (c) by SIAM. Unauthorized reproduction of this article is prohibited. 
Proof. Call $h_{1}(t):=\omega X_{t}(x)$ and $h_{2}(t):=X_{t}(\omega x)$. The second one satisfies the ODE

$$
h_{2}^{\prime}(t)=g\left(h_{2}(t)\right)
$$

while the first one satisfies

$$
h_{1}^{\prime}(t)=\omega g\left(X_{t}(x)\right)<g\left(\omega X_{t}(x)\right)=g\left(h_{1}(t)\right) .
$$

Since they have the same initial condition, this differential inequality implies $h_{1}(t)<$ $h_{2}(t)$ for all $t>0$.

Our main lower bound for the mitosis case is the following.

LEMMA 5.4 (lower bound for equal mitosis). Assume Hypotheses 1.1, 1.3, and 1.4 hold true with the mitosis kernel $p(z)=2 \delta_{\frac{1}{2}}(z)$. Let $\left(\mathcal{S}_{t}\right)_{t \geq 0}$ be the semigroup associated with (36). For any $\theta>0$ there exists $t_{0}=t_{0}(\theta)>0$ such that for all $t>t_{0}$ and $x_{0} \in(0, \theta]$ it holds that

$$
\mathcal{S}_{t} \delta_{x_{0}}(x) \geq C\left(t_{0}, \theta\right) \quad \text { for all } x \in I_{t},
$$

where $I_{t}$ is an open interval which depends on time $t$, and for some quantity $C=$ $C(t, \theta)$ depending only on $t$ and $\theta$.

Proof. Fix $\theta>0$ and take any $x_{0} \in(0, \theta]$. We follow the same strategy as in the proof of Lemma 5.1. Here the only different part is $\mathcal{A}(t, x)$. We consider the semigroup $\left(\mathcal{T}_{t}\right)_{t \geq 0}$ defined as in $(30)$ and $\left(\mathcal{S}_{t}\right)_{t \geq 0}$ defined as the semigroup associated with (36) with $\mathcal{A}(t, x)=4 B(2 x) m(t, 2 x)$. Using (34) we have

$$
\mathcal{T}_{t} \delta_{x_{0}}(2 x) \geq X_{t} \# \delta_{x_{0}}(2 x) e^{-C_{1} t}=\frac{1}{2} \delta_{\frac{1}{2} X_{t}\left(x_{0}\right)}(x) e^{-C_{1} t}
$$

for $C_{1}=C_{1}(\theta, t)$, increasing in $t$. we obtain

$$
\mathcal{A}(t, x) \geq 2 e^{-C_{1} t} B\left(X_{t}\left(x_{0}\right)\right) \delta_{\frac{1}{2} X_{t}\left(x_{0}\right)}(x) \text { for all } t>0 .
$$

We know that there exists some $x_{B}>0$ for which $B$ is bounded below by a positive quantity in each interval of the form $\left[x_{B}, R\right]$. Take $t_{B}>0$ such that for $t>t_{B}$ we have $X_{t}\left(x_{0}\right)>x_{B}$ for all $x_{0}>0$. Hence, for some $C_{2}=C_{2}(\theta, t)>0$, decreasing in $t$,

$$
\mathcal{A}(t, x) \geq C_{2} e^{-C_{1} t} \delta_{\frac{1}{2} X_{t}\left(x_{0}\right)}(x) \quad \text { for all } t>t_{B}
$$

Fix now any $t>t_{B}$. For $t_{B}<\tau<t$ we have

$$
\begin{aligned}
\mathcal{A}(\tau, x) & \geq C_{2}(\theta, \tau) e^{-C_{1}(\theta, \tau) \tau} \delta_{\frac{1}{2} X_{\tau}\left(x_{0}\right)}(x) \\
& \geq C_{2}(\theta, t) e^{-C_{1}(\theta, t) t} \delta_{\frac{1}{2} X_{\tau}\left(x_{0}\right)}(x)=: \tilde{C}_{2} e^{-\tilde{C}_{1} t} \delta_{\frac{1}{2} X_{\tau}\left(x_{0}\right)}(x)
\end{aligned}
$$

Hence using (29) we have

$$
\begin{aligned}
\mathcal{T}_{t-\tau} \mathcal{A}(\tau, x) & \geq \tilde{C}_{2} e^{-\tilde{C}_{1} t} \delta_{X_{t-\tau}\left(\frac{1}{2} X_{\tau}\left(x_{0}\right)\right)}(x) \exp \left(-\int_{0}^{t-\tau} c\left(X_{-s}(x)\right) \mathrm{d} s\right) \\
& \geq \tilde{C}_{2} e^{-2 \tilde{C}_{1} t} \delta_{X_{t-\tau}\left(\frac{1}{2} X_{\tau}\left(x_{0}\right)\right)}(x)
\end{aligned}
$$

Copyright (C) by SIAM. Unauthorized reproduction of this article is prohibited. 
for all $\tau \in\left(t_{B}, t\right)$. Define $F(\tau):=X_{t-\tau}\left(\frac{1}{2} X_{\tau}\left(x_{0}\right)\right)$, and notice that it is a strictly decreasing function, since Lemma 5.3 ensures that for $\tau_{1}<\tau_{2}$

$$
F\left(\tau_{2}\right)=X_{t-\tau_{2}}\left(\frac{1}{2} X_{\tau_{2}}\left(x_{0}\right)\right)<X_{t-\tau_{2}} X_{\tau_{2}-\tau_{1}}\left(\frac{1}{2} X_{\tau_{1}}\left(x_{0}\right)\right)=F\left(\tau_{1}\right) .
$$

By Lemma 5.2 we obtain

$$
\begin{aligned}
\int_{0}^{t} \mathcal{T}_{t-\tau} \mathcal{A}(\tau, x) \mathrm{d} \tau & \geq \int_{t_{B}}^{t} \mathcal{T}_{t-\tau} \mathcal{A}(\tau, x) \mathrm{d} \tau \geq \tilde{C}_{2} e^{-2 \tilde{C_{1}} t} \int_{t_{B}}^{t} \delta_{X_{t-\tau}\left(\frac{1}{2} X_{\tau}\left(x_{0}\right)\right)}(x) \mathrm{d} \tau \\
& \geq \tilde{C}_{2} e^{-2 \tilde{C_{1}} t}(F(\tau))^{\prime}(x) \mathbb{1}_{\mathcal{I}_{x_{0}}},
\end{aligned}
$$

where we define

$$
\mathcal{I}_{x_{0}}:=\left[\frac{1}{2} X_{t}\left(x_{0}\right), X_{t-t_{B}}\left(\frac{1}{2} X_{t_{B}}\left(x_{0}\right)\right)\right] .
$$

Again by Lemma 5.3 we see that this interval is nonempty. Since we need a bound which is independent of $x_{0}$, we consider the intersection of all these intervals as $x_{0}$ moves in the interval $(0, \theta)$. That intersection is

$$
\mathcal{I}_{t}:=\left[\frac{1}{2} X_{t}(\theta), X_{t-t_{B}}\left(\frac{1}{2} X_{t_{B}}(0)\right)\right] .
$$

Condition (13) shows that this interval is nonempty for $t$ large enough, since

$$
\frac{X_{t}(\theta)}{X_{t-t_{B}}\left(\frac{1}{2} X_{t_{B}}(0)\right)}=\frac{H^{-1}(t+\theta)}{H^{-1}\left(t-t_{B}+\frac{1}{2} X_{t_{B}}(0)\right)} \rightarrow 1 \quad \text { as } t \rightarrow+\infty .
$$

This gives the result.

6. Proof of the main result. We conclude by giving the proof of Theorem 1.3. It is a direct application of Harris's theorem 3.3. Hypotheses 3.2 and 3.3 need to be verified. We already verified Hypothesis 3.2 (Lyapunov condition) in section 4 (see the corollary given in each case); in fact, we have proved that given any $t_{0}>0$ we can satisfy Hypothesis 3.2 for any $t \geq t_{0}$ with constants $\gamma, K$ which are independent of $t$ ( since we can always take $\gamma:=e^{-C_{1} t_{0}}, K:=\tilde{C}$ ).

Regarding Hypothesis 3.3, the lower bounds we obtained in section 5 are for $m(t, x)$ which is a solution to (14). However we need to satisfy the minorization condition for $f(t, x)=\phi(x) m(t, x)$ since the equation on $f$ conserves mass; thus the associated semigroup is Markovian, and we may apply Harris's theorem to it. The equation satisfied by $f$ is

$$
\begin{array}{rlrl}
\frac{\partial}{\partial t} f(t, x)+\phi(x) \frac{\partial}{\partial x} & \left(\frac{g(x)}{\phi(x)} f(t, x)\right)+(B(x)+\lambda) f(t, x) & & \\
& =\phi(x) \int_{x}^{+\infty} \frac{B(y)}{y} p\left(\frac{x}{y}\right) f(t, y) \mathrm{d} y, & & t, x \geq 0, \\
m(t, 0) & =0, & t>0, \\
m(0, x) & =n_{0}(x), & & x>0 .
\end{array}
$$

We define $\left(\mathcal{F}_{t}\right)_{t \geq 0}$ as the semigroup associated with (37) or, alternatively, by the relationship

$$
\mathcal{F}_{t}\left(\phi n_{0}\right):=\phi \mathcal{S}_{t} n_{0}
$$

for any nonnegative measure $n_{0}$ such that $\phi n_{0}$ is a finite measure on $(0,+\infty)$.

Copyright $@$ by SIAM. Unauthorized reproduction of this article is prohibited. 
Lemma 6.1 (minorization condition for $f(t, x)$ ). We assume Hypotheses 1.1, 1.2, 1.3 , and 1.4 hold true. Let $\left(\mathcal{F}_{t}\right)_{t \geq 0}$ be the semigroup associated with (37). For any $0>\eta>\theta$ there exists $t_{0}=t_{0}(\eta, \theta)>0$ such that for all $t>t_{0}$ and $x_{0} \in[\eta, \theta]$ it holds that

$$
\mathcal{F}_{t} \delta_{x_{0}}(x) \geq \breve{C}(\eta, \theta, t) \quad \text { for all } x \in I_{t},
$$

where $I_{t}$ is an open interval which depends on time $t$, and for some quantity $\breve{C}=$ $\breve{C}(\eta, \theta, t)$ depending only on $\eta, \theta$, and $t$. If in addition we assume that

$$
\int_{0}^{1} \frac{1}{g(x)} \mathrm{d} x<+\infty
$$

then the above result also holds when taking $\eta=0$.

Proof. Let $\left(\mathcal{S}_{t}\right)_{t \geq 0}$ and $\left(\mathcal{F}_{t}\right)_{t \geq 0}$ be the semigroups associated with (14) and (37), respectively. Under the conditions of Lemma 5.1 we have a lower bound for $\mathcal{S}_{t} \delta_{x_{0}}(x) \geq$ $C(\eta, \theta, t)$. It immediately translates to a lower bound on $\mathcal{F}_{t}$ in all cases:

1. If $\int_{0}^{1} \frac{1}{g(x)} \mathrm{d} x=+\infty$, we know from [4] that $\phi(x)$ is bounded in each interval of the form $(0, \theta]$ (since it is continuous and tends to a positive constant at $x=0)$.

2. If $\int_{0}^{1} \frac{1}{g(x)} \mathrm{d} x=+\infty$, then since $\phi(x)$ is continuous there exist constants $\hat{C}_{1}(\eta, \theta), \hat{C}_{2}(\eta, \theta)>0$ such that $\hat{C}_{1} \leq \phi(y) \leq \hat{C}_{2}$ for all $y \in[\eta, \theta]$.

On the other hand, under the conditions of Lemma 5.4 we know again that $\phi(x)$ is bounded above and below by positive constants in each interval of the form $(0, \theta]$.

Therefore we obtain, for $x_{0} \in[\eta, \theta]$,

$$
\mathcal{F}_{t} \delta_{x_{0}}(x)=\frac{\phi(x)}{\phi\left(x_{0}\right)} \mathcal{S}_{t} \delta_{x_{0}}(x) \geq \frac{\hat{C}_{1}(\eta, \theta)}{\hat{C}_{2}(\eta, \theta)} C(\eta, \theta, t):=\breve{C}(\eta, \theta, t),
$$

allowing $\eta=0$ if $\int_{0}^{1} 1 / g<+\infty$.

Proof of Theorem 1.3. As remarked above, the semigroup $\left(\mathcal{F}_{t}\right)_{t \geq 0}$ satisfies the Lyapunov condition in Hypothesis 3.2 in all cases, for $t \geq 1$ with a weight $V$ and constants $\gamma, K$ which are independent of $t$. In order to satisfy Hypothesis 3.3 it is enough then to find any time $t \geq 1$ for which we have a uniform lower bound whenever the initial condition is a delta function supported on a region of the form

$$
\mathcal{C}:=\{x>0 \mid V(x) \leq R\}
$$

for some $R>2 K /(1-\gamma)$. Lemma 6.1 gives this in all cases. Notice that in the cases in which the lower bound is only available for $x_{0} \in[\eta, \theta]$ with $\eta>0$, the function $V$ we give in section 4 is unbounded at $x=0$, and thus the region $\mathcal{C}$ is contained in an interval of that form.

Explicit calculations for the self-similar fragmentation case. We recall that the so-called self-similar fragmentation equation corresponds to a linear growth rate $g(x)=x$, a monomial total fragmentation rate $B(x)=x^{b}, b>0$, and a selfsimilar kernel (here we take the homogeneous self-similar kernel $p(z) \equiv 2$ ). In that case, all the constants appearing in Harris's theorem can be quantified. This is due to the explicit expression $\phi(x)=x$ of the dual eigenfunction when $g(x)=x$. For the computations we choose, for instance, the parameters $k=0$ and $K=2$, which correspond to the Lyapunov function $V(x)=\left(x^{k}+x^{K}\right) / \phi(x)=1 / x+x$. We start 
with Hypothesis 3.2. Using that $B(x)=x^{b}$ we can make the proof of Lemma 4.1 more quantitative. Indeed the function $\Phi$ defined in (23) reads in the present case

$$
\Phi(x)=-\frac{1}{3} x^{b+1}+\frac{3}{2} x+x^{b-1}-\frac{1}{2} x^{-1} .
$$

Treating separately the cases $x \leq 1, x \geq 1$, and $b \leq 2, b \geq 2$, we can check that

$$
\Phi(x) \leq-\frac{1}{3} x^{b+1}+\frac{3}{2} x+x^{b-1} \leq 5\left(\frac{15}{2}\right)^{\frac{1}{b}+\frac{b}{2}}
$$

for all $x>0$. So Hypothesis 3.2 is verified for any $t_{0}>0$ with the constants

$$
\gamma=e^{-\frac{t_{0}}{2}} \quad \text { and } \quad K=10\left(\frac{15}{2}\right)^{\frac{1}{b}+\frac{b}{2}} .
$$

We now turn to Hypothesis 3.3. We choose

$$
R=\frac{4 K}{1-\gamma}
$$

and we notice that since $V(x)=1 / x+x$

$$
\mathcal{C}=\{x: V(x) \leq R\} \subset[1 / R, R] .
$$

For $\phi(x)=x$ and $p(z) \equiv 2,(37)$ reads

$$
\frac{\partial}{\partial t} f(t, x)+\frac{\partial}{\partial x}(x f(t, x))+B(x) f(t, x)=2 \int_{x}^{+\infty} B(y) f(t, y) \frac{x}{y} \mathrm{~d} y
$$

and we can prove directly on this equation, proceeding similarly as in Lemma 5.1, that for any $t_{0}>0$ and all $x_{0} \in[1 / R, R]$

$$
\mathcal{F}_{t_{0}} \delta_{x_{0}} \geq \alpha \nu
$$

with

$$
\nu(\mathrm{d} y)=\frac{2 e^{-2 t_{0}}}{R} \mathbb{1}_{\left[0, R e^{t_{0}}\right]}(y) y \mathrm{~d} y \quad \text { and } \quad \alpha=R^{b+3} t_{0} \exp \left(-2 R^{\gamma} \frac{e^{b t_{0}}}{b}\right) .
$$

We are now in position to apply Harris's theorem. Choosing in Theorem 3.3

$$
\alpha_{0}=\frac{\alpha}{2} \quad \text { and } \quad \gamma_{0}=\gamma+\frac{2 K}{R}
$$

we obtain

$$
\bar{\alpha}=\max \left\{1-\frac{\alpha}{2}, \frac{1-\gamma+\frac{1+\gamma}{2} \alpha}{1-\gamma+\alpha}\right\} .
$$

Choosing $t_{0}=2 \log 2$ we get

$$
\gamma=\frac{1}{2}, \quad R=80\left(\frac{15}{2}\right)^{\frac{1}{b}+\frac{b}{2}}, \quad \alpha=2 \log 2 R^{b+3} e^{-2(4 R)^{b} / b},
$$

and

$$
\bar{\alpha}=\max \left\{1-\frac{\alpha}{2}, 1-\frac{\alpha}{2(1+2 \alpha)}\right\}=1-\frac{\alpha}{2(1+2 \alpha)} .
$$

This proves that we can choose $\rho$ as in (16).

Copyright $@$ by SIAM. Unauthorized reproduction of this article is prohibited. 


\section{REFERENCES}

[1] M. Adimy and L. Pujo-Menjouet, Asymptotic behavior of a singular transport equation modelling cell division, Discrete Contin. Dyn. Syst. Ser. B, 3 (2003), pp. 439-456.

[2] O. ARINO, Some spectral properties for the asymptotic behavior of semigroups connected to population dynamics, SIAM Rev., 34 (1992), pp. 445-476.

[3] F. Baccelli, D. R. McDonald, And J. Reynier, A mean-field model for multiple TCP connections through a buffer implementing red, Perform. Eval., 49 (2002), pp. 77-97.

[4] D. Balagué, J. A. Cañizo, and P. Gabriel, Fine asymptotics of profiles and relaxation to equilibrium for growth-fragmentation equations with variable drift rates, Kinet. Relat. Models, 6 (2013), pp. 219-243.

[5] J. Banasiak and L. Arlotti, Perturbations of Positive Semigroups with Applications, Springer, London, 2006.

[6] J. Banasiak, W. Lamb, And P. Laurençot, Analytic Methods for Coagulation-Fragmentation Models. Volume I, CRC, Boca Raton, FL, 2019.

[7] J. BAnAsiak, K. PichóR, And R. Rudnicki, Asynchronous exponential growth of a general structured population model, Acta Appl. Math., 119 (2012), pp. 149-166.

[8] V. Bansaye, B. Cloez, And P. Gabriel, Ergodic behavior of non-conservative semigroups via generalized Doeblin's conditions, Acta Appl. Math., 166 (2020), pp. 29-72, https://doi.org/ 10.1007/s10440-019-00253-5.

[9] V. Bansaye, B. Cloez, P. Gabriel, and A. Marguet, A Non-Conservative Harris Ergodic Theorem, preprint, https://arxiv.org/abs/1903.03946 (2019).

[10] J.-B. Bardet, A. Christen, A. Guillin, F. Malrieu, and P.-A. Zitt, Total variation estimates for the TCP process, Electron. J. Probab., 18 (2013), pp. 1-21.

[11] G. I. BeLL, Cell growth and division. III. Conditions for balanced exponential growth in a mathematical model, Biophys. J., 8 (1968), pp. 431-444.

[12] G. I. Bell And E. C. Anderson, Cell growth and division. I. A mathematical model with applications to cell volume distributions in mammalian suspension cultures, Biophys. J., 7 (1967), pp. 329-351.

[13] E. Bernard, M. Doumic, And P. Gabriel, Cyclic asymptotic behaviour of a population reproducing by fission into two equal parts, Kinet. Relat. Models, 12 (2019), pp. 551-571.

[14] E. Bernard AND P. Gabriel, Asymptotic behavior of the growth-fragmentation equation with bounded fragmentation rate, J. Funct. Anal., 272 (2017), pp. 3455-3485.

[15] E. Bernard And P. Gabriel, Asynchronous exponential growth of the growth-fragmentation equation with unbounded fragmentation rate, J. Evol. Equ., 20 (2020), pp. 375-401.

[16] J. BerToIn, The asymptotic behavior of fragmentation processes, J. Eur. Math. Soc. (JEMS), 5 (2003), pp. 395-416.

[17] J. Bertoin, On a Feynman-Kac approach to growth-fragmentation semigroups and their asymptotic behaviors, J. Funct. Anal., 277 (2019), 108270.

[18] J. Bertoin And A. R. Watson, Probabilistic aspects of critical growth-fragmentation equations, Adv. Appl. Probab., 48 (2016), pp. 37-61.

[19] J. Bertoin And A. R. WAtson, A probabilistic approach to spectral analysis of growthfragmentation equations, J. Funct. Anal., 274 (2018), pp. 2163-2204.

[20] J. Bertoin AND A. R. WATSOn, The strong Malthusian behavior of growth-fragmentation processes, Ann. H. Lebesgue, 3 (2020), pp. 795-823, https://doi.org/10.5802/ahl.46.

[21] F. Bouguet, A probabilistic look at conservative growth-fragmentation equations, in Séminaire de Probabilités XLIX, Springer, Cham, Switzerland, 2018, pp. 57-74.

[22] F. B. Brikci, J. Clairambault, and B. Perthame, Analysis of a molecular structured population model with possible polynomial growth for the cell division cycle, Math. Comput. Modelling, 47 (2008), pp. 699-713.

[23] J. Broda, A. Grigo, And N. P. Petrov, Convergence rates for semistochastic processes, Discrete Contin. Dyn. Syst. Ser. B, 24 (2019), pp. 109-125.

[24] J. A. Cañizo, J. A. Carrillo, And S. Cuadrado, Measure solutions for some models in population dynamics, Acta Appl. Math., 123 (2013), pp. 141-156, https://doi.org/10.1007/ s10440-012-9758-3.

[25] J. A. CAÑIZO AND H. YoldaŞ, Asymptotic behaviour of neuron population models structured by elapsed-time, Nonlinearity, 32 (2019), pp. 464-495.

[26] M. J. CÁCERES, J. A. CAÑzo, and S. Mischler, Rate of convergence to self-similarity for the fragmentation equation in $L^{1}$ spaces, Commun. Appl. Ind. Math., 1 (2010), pp. 299-308.

[27] M. J. CÁCERes, J. A. CAÑIzo, AND S. Mischler, Rate of convergence to an asymptotic profile for the self-similar fragmentation and growth-fragmentation equations, J. Math. Pures Appl. (9), 96 (2011), pp. 334-362.

Copyright (c) by SIAM. Unauthorized reproduction of this article is prohibited. 
[28] V. Calvez, N. Lenuzza, D. Oelz, J.-P. Deslys, P. Laurent, F. Mouthon, and B. Perthame, Size distribution dependence of prion aggregates infectivity, Math. Biosci., 217 (2009), pp. 88-99.

[29] B. CAVALLI, A probabilistic view on the long-time behaviour of growth-fragmentation semigroups with bounded fragmentation rates, ESAIM:PS, 25 (2021), pp. 258-285, https://doi.org/10. $1051 / \mathrm{ps} / 2021008$.

[30] B. CavalLi, On a family of critical growth-fragmentation semigroups and refracted Lévy processes, Acta Appl. Math., 166 (2020), pp. 161-186.

[31] D. Chafaï, F. Malrieu, and K. Paroux, On the long time behavior of the TCP window size process, Stochastic Process. Appl., 120 (2010), pp. 1518-1534, https://doi.org/10.1016/j. spa.2010.03.019.

[32] B. ClOEZ, Limit theorems for some branching measure-valued processes, Adv. Appl. Probab., 49 (2017), pp. 549-580.

[33] B. Cloez AND P. Gabriel, On an irreducibility type condition for the ergodicity of nonconservative semigroups, C. R. Math. Acad. Sci. Paris, 358 (2020), pp. 733-742, https: //doi.org/10.5802/crmath.92.

[34] T. Dȩbiec, M. Doumic, P. Gwiazda, and E. Wiedemann, Relative entropy method for measure solutions of the growth-fragmentation equation, SIAM J. Math. Anal., 50 (2018), pp. 58115824.

[35] O. Diekmann, H. J. A. M. Heijmans, and H. R. Thieme, On the stability of the cell size distribution, J. Math. Biol., 19 (1984), pp. 227-248.

[36] J. L. DooB, Conditional Brownian motion and the boundary limits of harmonic functions, Bull. Soc. Math. France, 85 (1957), pp. 431-458.

[37] M. Doumic And M. Escobedo, Time asymptotics for a critical case in fragmentation and growth-fragmentation equations, Kinet. Relat. Models, 9 (2016), pp. 251-297.

[38] M. Doumic Jauffret And P. Gabriel, Eigenelements of a general aggregation-fragmentation model, Math. Models Methods Appl. Sci., 20 (2010), pp. 757-783.

[39] G. Dumont And P. Gabriel, The mean-field equation of a leaky integrate-and-fire neural network: Measure solutions and steady states, Nonlinearity, 33 (2020), pp. 6381-6420.

[40] K.-J. Engel And R. NAGel, One-Parameter Semigroups for Linear Evolution Equations, Grad. Texts in Math. 194, Springer, New York, 2000.

[41] H. Engler, J. Prüss, And G. F. WebB, Analysis of a model for the dynamics of prions. II, J. Math. Anal. Appl., 324 (2006), pp. 98-117.

[42] M. Escobedo, S. Mischler, and M. Rodriguez Ricard, On self-similarity and stationary problem for fragmentation and coagulation models, Ann. Inst. H. Poincaré Anal. Non Linéaire, 22 (2005), pp. 99-125, https://doi.org/10.1016/j.anihpc.2004.06.001.

[43] P. Gabriel, Global stability for the prion equation with general incidence, Math. Biosci. Eng., 12 (2015), pp. 789-801.

[44] P. GABRIEL, Measure solutions to the conservative renewal equation, ESAIM, Proc. Surveys, 62 (2018), pp. 68-78.

[45] P. Gabriel and H. Martin, Periodic asymptotic dynamics of the measure solutions to an equal mitosis equation, Ann. H. Lebesgue (2021), accepted.

[46] P. Gabriel AND F. SAlvarani, Exponential relaxation to self-similarity for the superquadratic fragmentation equation, Appl. Math. Lett., 27 (2014), pp. 74-78.

[47] G. Greiner And R. NAGel, Growth of cell populations via one-parameter semigroups of positive operators, in Mathematics Applied to Science, Academic Press, Boston, 1988, pp. 79-105.

[48] M. Hairer, Convergence of Markov Processes, Lecture notes, 2016.

[49] M. Hairer and J. C. Mattingly, Yet another look at Harris' ergodic theorem for Markov chains, in Seminar on Stochastic Analysis, Random Fields and Applications VI, Centro Stefano Franscini, Ascona (Ticino), Switzerland, 2008, Birkhäuser, Basel, 2011, pp. 109117.

[50] A. J. Hall and G. C. WaKe, A functional-differential equation arising in modelling of cell growth, ANZIAM J., 30 (1989), pp. 424-435, https://doi.org/10.1017/S0334270000006366.

[51] A. J. Hall and G. C. WaKe, Functional differential equations determining steady size distributions for populations of cells growing exponentially, ANZIAM J., 31 (1990), pp. 434-453.

[52] T. E. HARRIS, The existence of stationary measures for certain Markov processes, in Proceedings of the Third Berkeley Symposium on Mathematical Statistics and Probability, 1954-1955, vol. II, University of California Press, Berkeley, 1956, pp. 113-124.

[53] H. J. A. M. HeiJmans, An eigenvalue problem related to cell growth, J. Math. Anal. Appl., 111 (1985), pp. 253-280.

[54] P. Laurençot and B. Perthame, Exponential decay for the growth-fragmentation/cell-division equations, Commun. Math. Sci., 7 (2009), pp. 503-510.

Copyright (c) by SIAM. Unauthorized reproduction of this article is prohibited. 
[55] E. LeIS AND C. WALKER, Existence of global classical and weak solutions to a prion equation with polymer joining, J. Evol. Equ., 17 (2017), pp. 1227-1258, https://doi.org/10.1007/ s00028-016-0379-6.

[56] E. D. MCGrady AND R. M. ZiFF, "Shattering" transition in fragmentation, Phys. Rev. Lett., 58 (1987), pp. 892-895, https://doi.org/10.1103/PhysRevLett.58.892.

[57] J. A. Metz and O. Diekmann, The Dynamics of Physiologically Structured Populations, Lecture Notes in Biomath. 68, Springer, Berlin, 2014.

[58] S. P. Meyn and R. L. Tweedie, Markov Chains and Stochastic Stability, Comm. Control Eng. Ser., Springer, London, 1993, https://doi.org/10.1007/978-1-4471-3267-7.

[59] P. Michel, Existence of a solution to the cell division eigenproblem, Math. Models Methods Appl. Sci., 16 (2006), pp. 1125-1153, https://doi.org/10.1142/S0218202506001480.

[60] P. Michel, S. Mischler, and B. Perthame, General relative entropy inequality: An illustration on growth models, J. Math. Pures Appl. (9), 84 (2005), pp. 1235-1260, https: //doi.org/10.1016/j.matpur.2005.04.001.

[61] S. Mischler And J. Scher, Spectral analysis of semigroups and growth-fragmentation equations, Ann. Inst. Henri Poincaré Anal. Non Linéaire, 33 (2016), pp. 849-898.

[62] K. Pakdaman, B. Perthame, and D. Salort, Adaptation and fatigue model for neuron networks and large time asymptotics in a nonlinear fragmentation equation, J. Math. Neurosci., 4 (2014), 14, https://doi.org/10.1186/2190-8567-4-14.

[63] B. Perthame, Transport Equations in Biology, Front. Math., Springer, Basel, 2006, https: //doi.org/10.1007/978-3-7643-7842-4.

[64] B. Perthame And L. Ryzhik, Exponential decay for the fragmentation or cell-division equation, J. Differential Equations, 210 (2005), pp. 155-177.

[65] R. Rudnicki and M. Tyran-Kamińska, Piecewise Deterministic Processes in Biological Model, Springer, Cham, Switzerland, 2017.

[66] J. W. Sinko And W. Streifer, A model for populations reproducing by fission, Ecology, 52 (1971), pp. 330-335.

[67] D. W. Stroock, An Introduction to Markov Processes, Grad. Texts in Math. 230, Springer, New York, 2013.

[68] B. van Brunt, A. Almalki, T. Lynch, And A. A. Zaidi, On a cell division equation with a linear growth rate, ANZIAM J., 59 (2018), pp. 293-312.

[69] A. A. Zaidi, B. van Brunt, And G. C. Wake, Solutions to an advanced functional partial differential equation of the pantograph type, Proc. A, 471 (2015), 20140947.

Copyright (C) by SIAM. Unauthorized reproduction of this article is prohibited. 